\title{
LITHOLOGICAL AND FORAMINIFERAL CHARACTERISTICS OF SHOREFACE AND SHALLOW SHELF FACIES OFF BOGUE BANKS, NC
}

\author{
by \\ Lillian Howie \\ A Senior Honors Project Presented to the \\ Honors College \\ East Carolina University \\ In Partial Fulfillment of the \\ Requirements for \\ Graduation with Honors \\ by \\ Lillian Howie \\ Greenville, NC \\ May 2018
}

Approved by:

Dr. Stephen Culver

Department of Geological Sciences, Thomas Harriot College of Arts and Sciences 


\section{ABSTRACT}

Fossil foraminiferal assemblages are used by paleontologists to determine the depositional environment of the strata in which the assemblages are found. This allows for the reconstruction of past environments and climates working under the assumption that specific foraminiferal assemblages are diagnostic to the depositional environment. However, foraminiferal assemblages of several coastal subenvironments (e.g., beach, shoreface, ebb tide delta, inner shelf) have yet to be extensively studied. In this study, Holocene sediments from vibracores taken off the coast of Bogue Banks, NC, were analyzed for their lithology and foraminiferal assemblages to study the differences before shoreface and inner shelf environments. Two $3 \mathrm{~m}$ vibracores from each environment were logged using a method that is independent from composition, and samples of sediment from Holocene units were taken for foraminiferal analysis. Sand and mud content were determined by sieving. The 63-710 micron fraction of the samples were floated in a sodium polytungstate solution to concentrate foraminiferal tests. Approximately 100 specimens were randomly picked from each sample and the relative percentages of three major foraminiferal taxonomic groups were recorded. In shelf sediment samples, assemblages comprised $95 \%$ to $100 \%$ Rotaliina. By comparison, in shoreface sediment samples, assemblages comprised $85 \%$ to $90 \%$ Rotaliina, with $10 \%$ to $15 \%$ Miliolina. These results suggest that a potential method for distinguishing the two subenvironments could be found in the presence of absence of genera within the suborder Miliolina. 


\section{ACKNOWLEDGEMENTS}

We acknowledge the generous donors of the American Chemical Society Petroleum Research Fund for the project funding. We also acknowledge Greg "rudi" Rudolph for providing the cores, and Caroline Smith for training in lab procedures. 


\section{TABLE OF CONTENTS}

ACKNOWLEDGEMENTS




\section{LIST OF FIGURES}

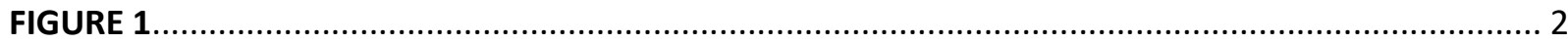

(A) Map of the study area, showing the locations of Swansboro, Bogue, and Emerald Isle in relation to Bogue Inlet on the left and the area from which cores were taken (marked in red). Satellite imagery from Google Map data, 2016. (B) Inset of the area from which cores were taken, showing the location of Bogue Inlet Fishing Pier at the top. Satellite imagery from Google Map data, 2016.

FIGURE 2 . 5

(A - D) Computer-generated core logs for 3 meters of cores $Y-69, Y-70, Y-91$, and $Y-103$ alongside graphic interpretations of data for each sample. The data includes, from left to right, \% fossil, number of species, calculated specimens per gram of sediment, number of species, alpha, and relative percentages of the most abundant species in each core.

FIGURE 3... 9

Ternary diagram showing the relative abundance of the three foraminiferal suborders, Rotaliina, Miliolina, and Textulariina, with data points colored by core number for identification. 


\section{INTRODUCTION}

The fossilized remains of foraminifera have long been used in paleoenvironmental reconstruction for many different environments and time frames, and are essential to many industries that depend on the understanding of ancient environments and their impacts on sediment formation and deposition. A difficult task in interpreting a coastal stratigraphic record is distinguishing between certain coastal subenvironments such as coastal dune, shoreface, shallow shelf, and ebb tide delta (Culver, 2014). Because these subenvironments have very similar lithologies and sedimentary structures, trying to interpret them becomes problematic, and other factors need to be examined. A common method of examining and reconstructing past environments is the use of foraminiferal assemblages, however sufficient research on the use of foraminiferal assemblages in distinguishing between subenvironments has not yet been done. Little foraminiferal research has been done on the seaward side of barrier islands such as those found off the coast of North Carolina, and the integration of foraminiferal and sedimentological studies in this area is also lacking. The purpose of this project was to combine sedimentological and foraminiferal studies of cores taken from off the coast of North Carolina in order to document and help define the foraminiferal assemblages found in the Shoreface and Shallow Shelf environments. 


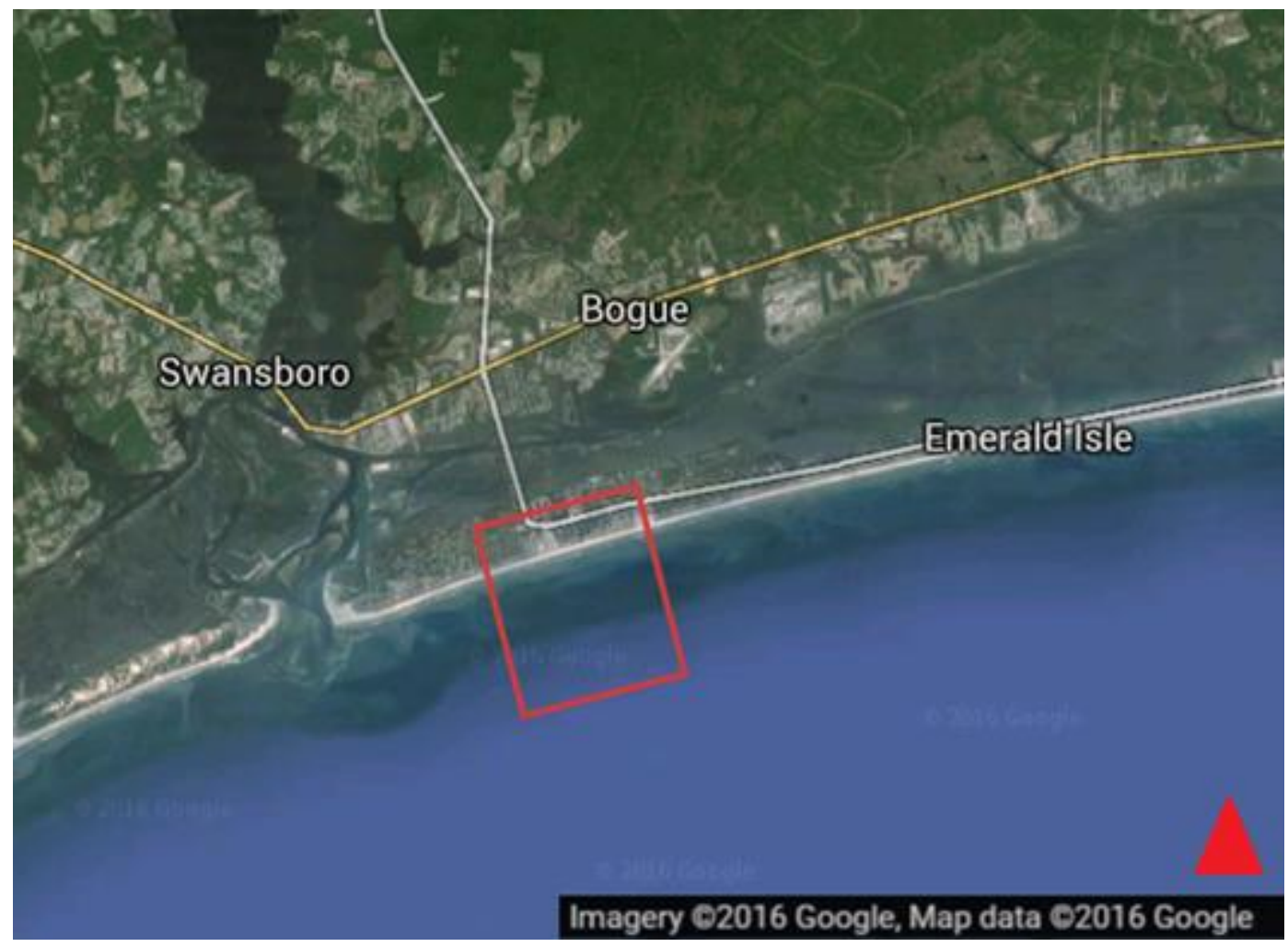

Fig. 1A: Map of the surveyed location off Bogue Banks, North Carolina. See fig. 1 B for specific core locations.

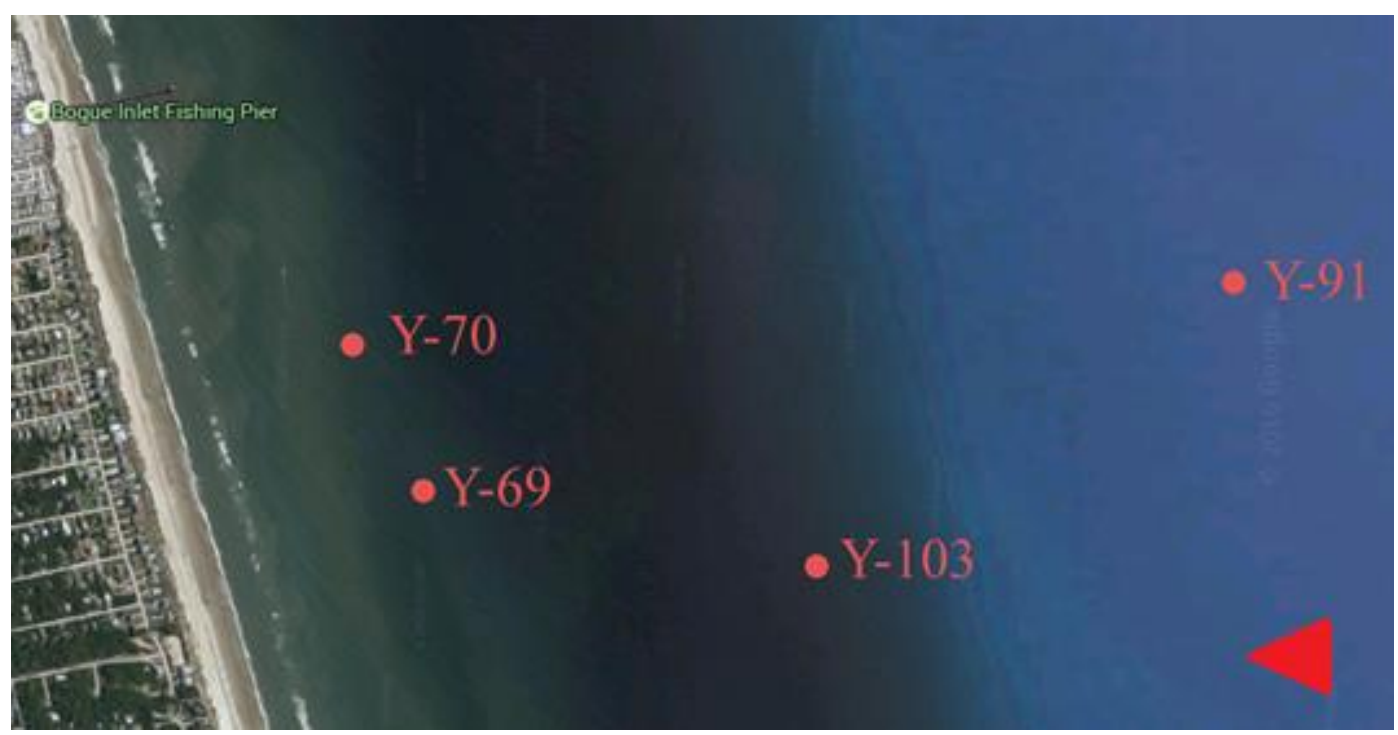

Fig. 1B: Inset with locations of individual cores studied. These cores were taken between 10-20 meters water depth. 


\section{PREVIOUS WORK}

In 1971, an investigation into the foraminiferal distribution off the north Carolina coast was published by Detmar Schnitker. This study surveyed foraminifera in Near-shore, Central Shelf, and Shelf Edge environments and used the data to construct maps showing the distribution of species. The study found that there was a major boundary located at Cape Hatteras, south of which the species found are similar to those from Florida and the Gulf of Mexico (Schnitker, 1971). Because the location from which the cores were taken is south of Cape Hatteras, it was reasonable to assume that the characteristics of the assemblages found in Schnitker's study can be applied to this area. However, Schnitker's study was very limited in the samples taken near-shore, leaving it with a poorly-defined picture of the near shore foraminiferal assemblages (1971). Workman's 1981 thesis investigated benthic foraminifera living off the along the near-shore coast of North Carolina in Onslow Bay and just off Nags Head. Because of the geography of the North Carolina coast and the two distinct water masses that characterize it, two foraminiferal assemblages exist along the coast south of Cape Hatteras, where more diverse subtropical foraminifera are located, and north of Cape Hatteras, where a less diverse population of temperate foraminifera are located (Workman, 1981). The Onslow Bay samples yielded abundant species such as Ammonia tepida, Elphidium excavatum, and members of the genus Quinqueloculina, the latter of which became more common in samples farther offshore (Workman, 1981). A more recent study was done in 2009 by Smith et. al. examining foraminiferal assemblages in Holocene Flood Tide Deltas. This study characterized five biofacies using foraminiferal data, two of which were dominated by calcareous marine or estuarine foraminifera, and three of which were dominated by agglutinated marsh species (Smith et. al., 2009). Carolina Smith's 2015 thesis studied foraminiferal assemblages found in the Ocracoke Flood-Tide Delta, finding four biofacies dominated by species such as Elphidium excavatum and Ammonia parkinsoniana. These facies were predominantly fine-to-medium grained sand (Smith, 2015). 


\section{METHODS}

As part of a sand resource investigation, a suite of vibracores was taken in 2011 from environments off Bogue Banks, North Carolina. These cores (fig. \#) represent shoreface and shallow shelf facies between 10 and 20 meters water depth. From these cores, four were selected (fig. \#), two to represent each subenvironment. These cores were logged to define lithofacies using the sedimentological characteristics of the contents and from the determined lithofacies, a probable Holocene-Pleistocene boundary could also be defined. Using this boundary as a guide, samples were taken from each unit determined to be Holocene in age to be subjected to foraminiferal analysis. Graphic logs were drawn up using the protocol established by Farrell et al. (2013).

A total of 16 samples $\left(27 \mathrm{~cm}^{3}\right.$ each) were taken from the four cores to represent each of the Holocene units. These samples were dried and weighed, then were disaggregated, sieved to separate sand and gravel portions, dried, and weighed again. Using the weights of the final dried samples, the sand to mud ratio of the samples was determined.

Because all samples were sand-rich, the samples were floated in sodium polytungstate to concentrate the foraminiferal tests before picking. After being floated, the samples were split with a microsplitter if needed and spread evenly onto a picking tray. A random number sheet was used to randomly select approximately 100 specimens from each sample. These specimens were identified to the species level using published and unpublished literature, and were also classified as recent or fossil based on their preservation. 


\section{RESULTS}

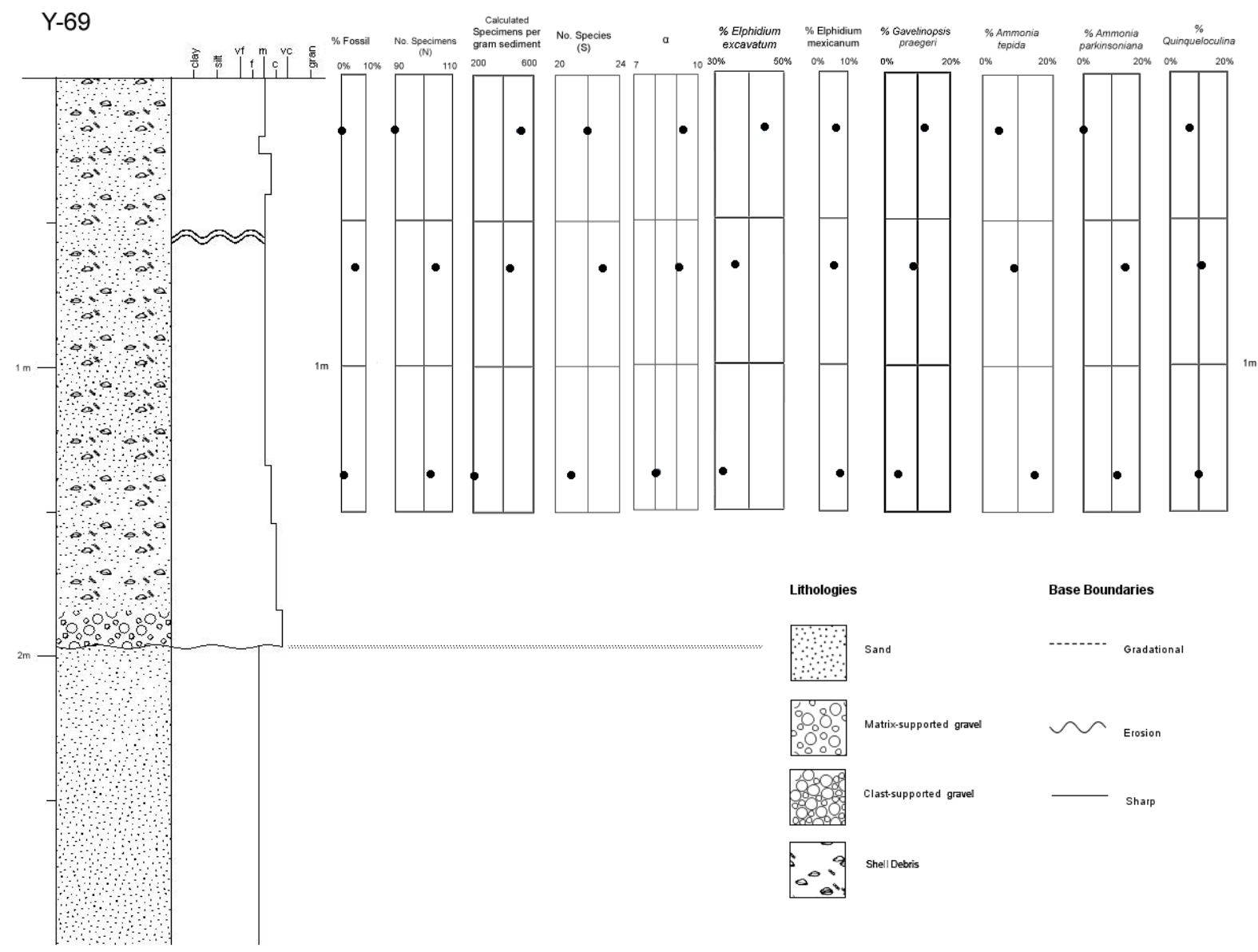

Fig. 2A: Core log and foraminiferal data for Y-69, showing lithological units alongside data for \% fossil, number of species, calculated specimens per gram of sediment, number of species, alpha, and relative percentages of the most abundant species. Refer to the key for core lithologies. 


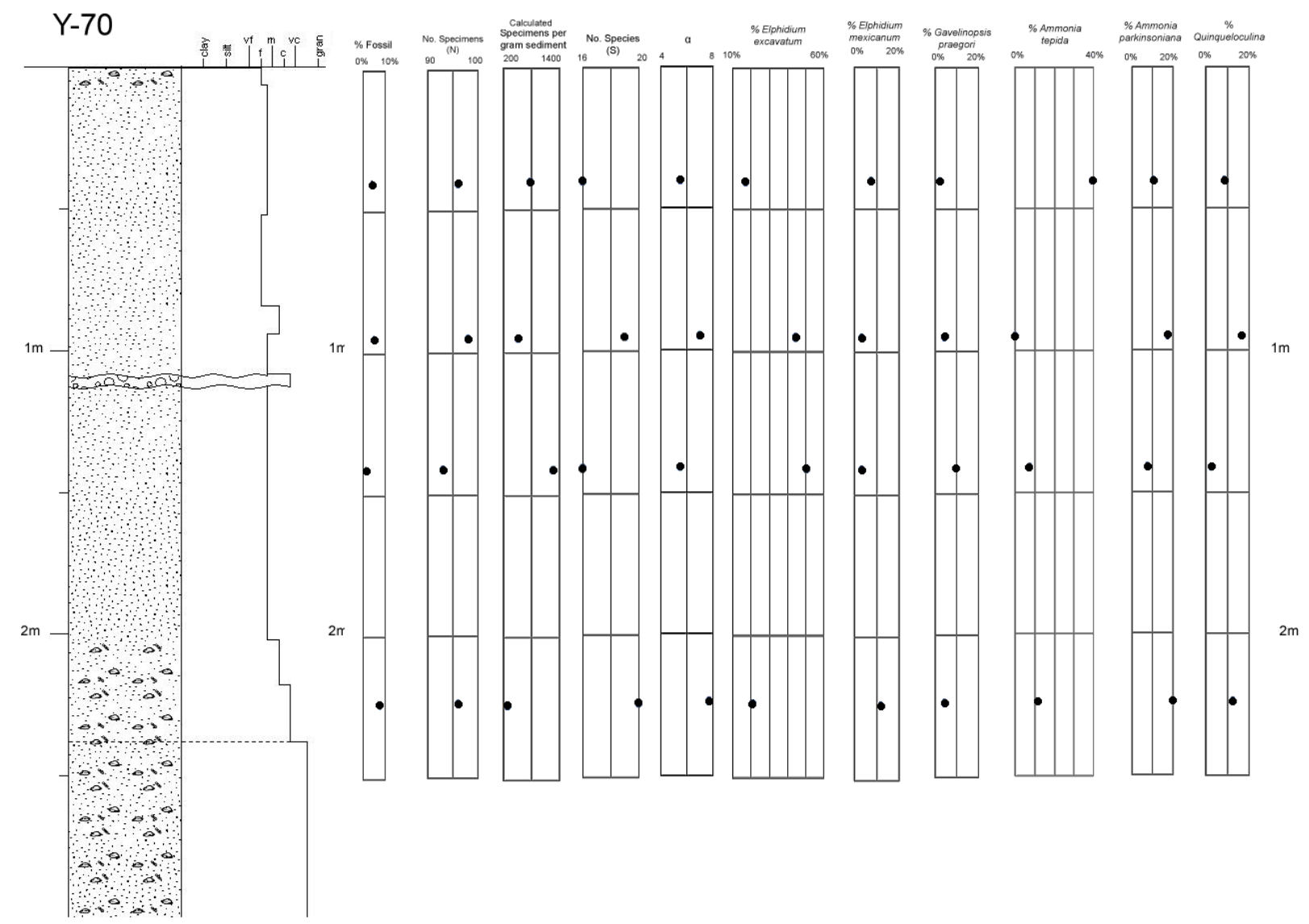

Fig. 2B: Core log and foraminiferal data for $Y-70$, showing lithological units alongside data for \% fossil, number of species, calculated specimens per gram of sediment, number of species, alpha, and relative percentages of the most abundant species. Refer to the key in Fig. 2.1 for core lithologies. 


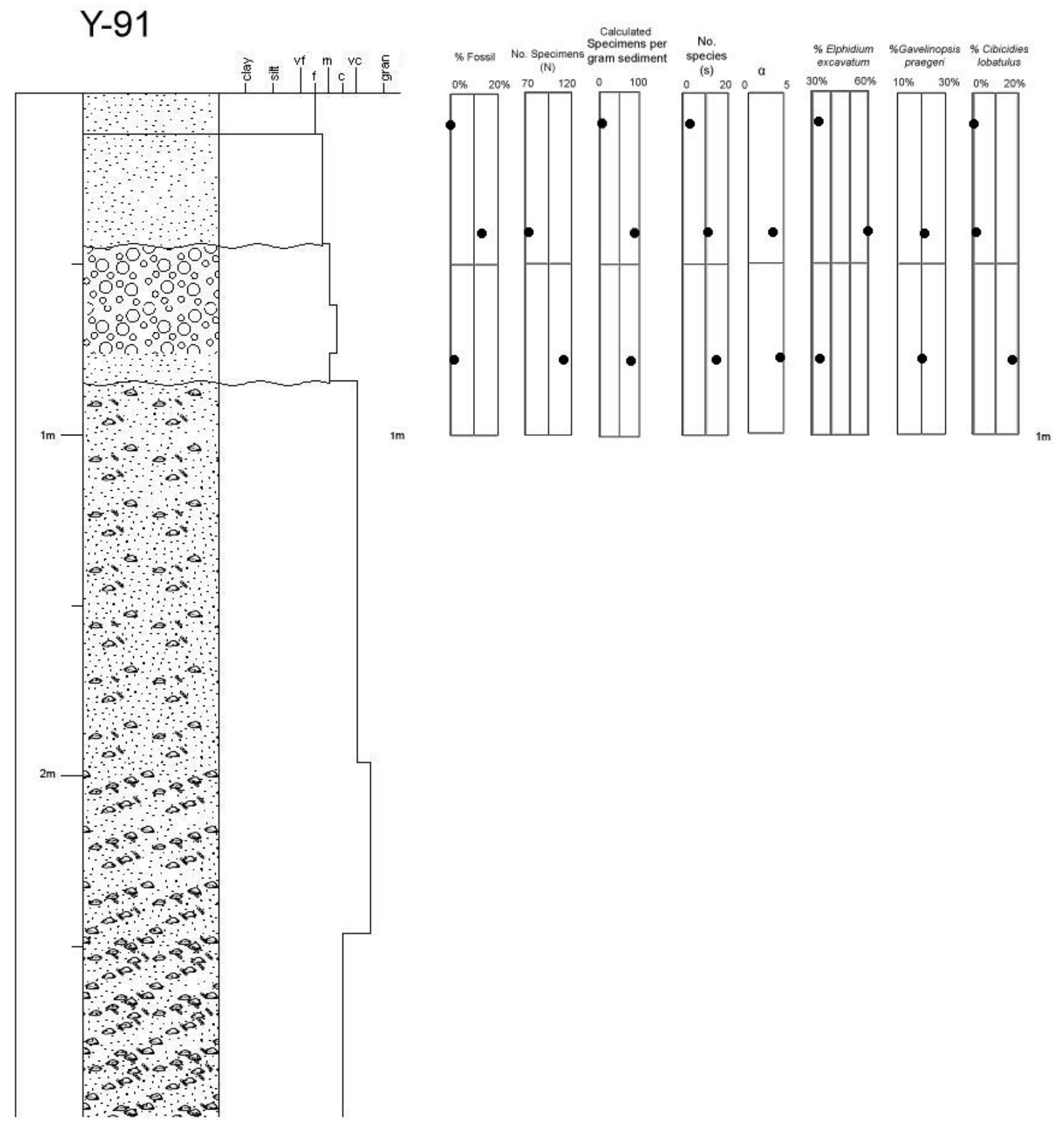

Fig. 2C: Core log and foraminiferal data for Y-91, showing lithological units alongside data for \% fossil, number of species, calculated specimens per gram of sediment, number of species, alpha, and relative percentages of the most abundant species. Refer to the key in Fig. 2.1 for core lithologies. 


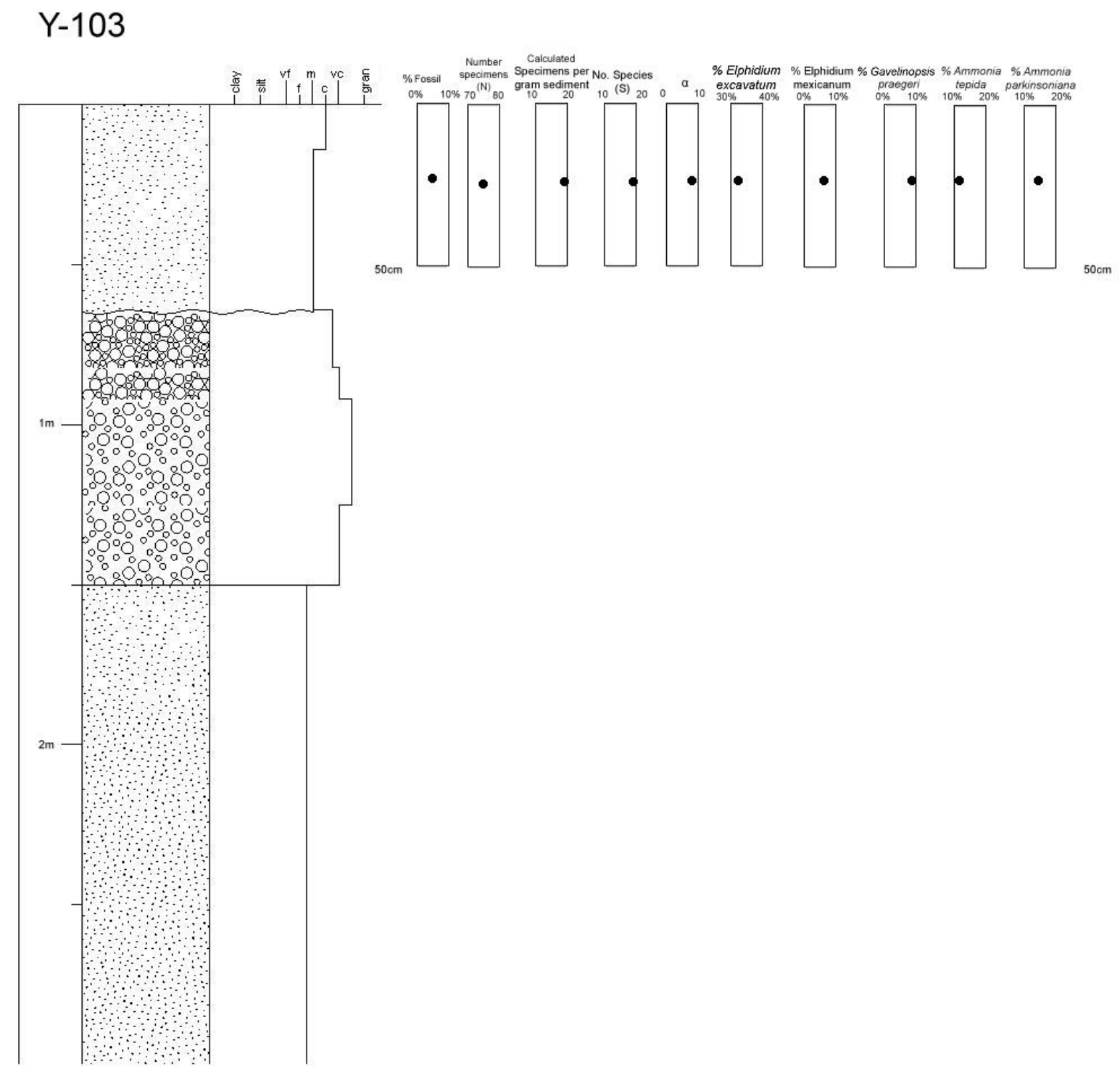

Fig. 2D: Core log and foraminiferal data for Y-103, showing lithological units alongside data for \% fossil, number of species, calculated specimens per gram of sediment, number of species, alpha, and relative percentages of the most abundant species. Refer to the key in Fig. 2.1 for core lithologies. 


\section{Ternary Diagram}

Miliolina

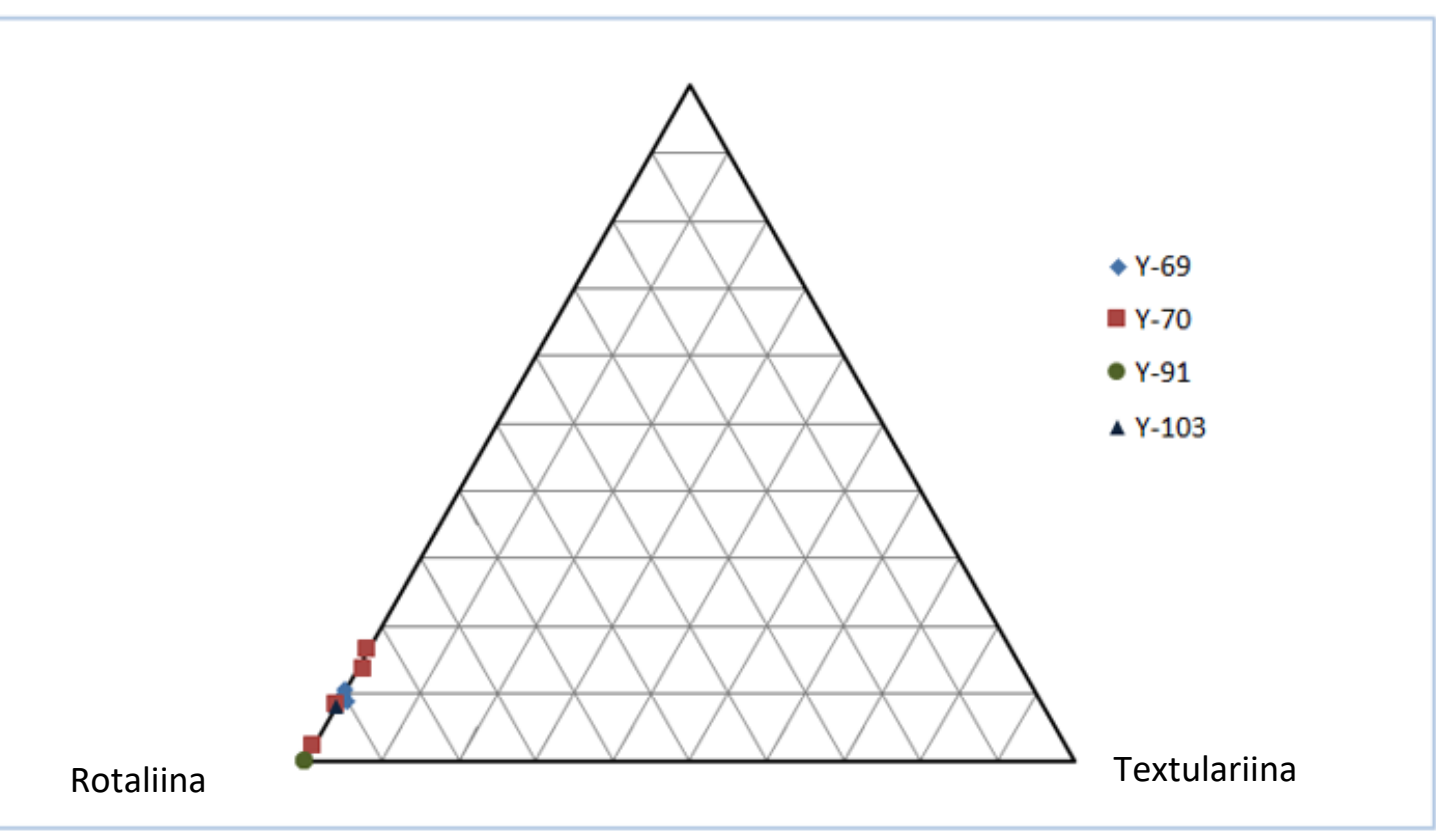

Fig. 3: Ternary diagram showing relative percentages of foraminiferal suborders in each sample, separated by core.

\section{DISCUSSION}

The ternary diagram (fig. 3) showed that all samples were dominated by small rotaliina, with few miliolina and almost no textulariina. Examination of the trends in the overall core data reveals that miliolina are more abundant closer to the shore, and decrease in abundance farther from shore until they are completely absent. The studies done by Culver, Abbene, and Vance showed that the source of the miliolina is likely the brackish water on the other side of the barrier islands, and those present in the shoreface and shelf facies have been carried there through inlets. Michael Twarog's work, done at the same time as this study, showed large percentages of miliolina in inlet throat and ebb tide delta environments. However, the discovery of miliolina, particularly Quinqueloculina appearing more abundantly near-shore than farther off-shore is the opposite of what was found in Onslow Bay in the Workman (1981) thesis. This discrepancy provides a topic for further study, as the species of 
Quinqueloculina were the only specimens that allowed for differentiation between the shoreface and the shelf samples.

The two shoreface cores showed very similar lithologies and foraminiferal assemblages. In core Y-69, species diversity steadily increased up-core, while the diversity values in Y-70 did not display the same pattern. Similarly, the calculated number of specimens per gram of sediment increased up-core in Y-69, but did not show a steady pattern in Y-70. In both cores, examination of species data showed an inverse relationship between the genera Elphidium and Gavelinopsis and the genus Ammonia. This suggests fluctuations in water salinity, with Ammonia preferring higher-salinity environments than Elphidium and Gavelinopsis. In both shoreface cores, higher values of species richness and specimens per gram of sediment are usually found in samples that have higher relative percentages of Elphidium and lower relative percentages of Ammonia.

Lithologically, the two shelf cores were extremely different from one another, with HolocenePleistocene boundaries determined to be much closer to the surface than those of the shoreface cores. Much of the sediment in core $\mathrm{Y}-103$, in fact, showed terrestrial depositional environments rather than marine. Because core Y-103 yielded only one sample of foraminiferal species, it is difficult to properly compare the patterns found in the shelf cores. However, the relationship between the species mentioned above were the same, with higher relative percentages of Elphidium correlated with lower relative percentages of foraminifera such as Cibicides.

In the end, the most abundant foraminifera found in both shoreface and shelf cores were the same: the assemblages were dominated by Elphidium excavatum, Elphidium mexicanum, Gavelinopsis praegeri, Ammonia parkinsoniana, and Ammonia tepida. Comparing the most abundant species in each sample did not produce a discernable difference between samples of different subenvironments. However, a difference was found in the abundance of genera Quniqueloculina and Triloculina, which were present in 
shoreface cores, but were either rare or absent in shelf cores. This difference suggests a potential method for distinguishing between shoreface and shallow shelf facies which must be investigated further to determine reliability. 


\section{REFERENCES}

Abbene, I.J., Culver, S.J., Corbett, D.R., Buzas, M.A., Tully, L.S., 2006. Distribution of framinifera in Pamlico Sound, North Carolina over the past century. Journal of Foraminiferal Research, 36, 136151.

Grand Pre, C. A., \& East Carolina University. Department of Geology, 2006. Holocene paleoenvironmental change in Pamlico Sound, North Carolina: foraminiferal and stable isotopic evidence.

Hayek, L. C., and Buzas, M. A., 1997. Surveying Natural Populations. Columbia University Press, New York.

Loeblich, A.R. Jr., Tappan, H, 1964. Treatise on Invertebrate Paleontology: Protista 2: Sarcodina, chiefly "Thecamoebians" and Foraminiferida. The University of Kansas Press and The Geological Society of America.

Schnitker, D., 1971, Distribution of foraminifera on the North Carolina continental shelf: Tulane Studies in Geology and Paleontology, v. 8.

Smith, C.F., 2015. Holocene evolution of the Ocracoke flood-tide delta region, Outer Banks, North Carolina. M.S. Thesis, East Carolina University, Greenville, NC.

Smith, C.G., Culver, S.J., Mallinson, D.J., Riggs, S.R., Corbett, D.R., 2009. Recognizing former flood-tide deltas in the Holocene stratigraphic record from the Outer Banks, North Carolina, USA. Stratigraphy, 6.

Todd, R., Low D., 1981. Marine flora and fauna of the northeastern United States: Protozoa: Sarcodina: benthic foraminifera. NOAA Tech. Rep. NMFS CIRC.

Vance, D. J., 2004, Modern and historic trends in foraminiferal distributions and sediment dynamics in the Albemarle estuarine system, North Carolina. M.S. Thesis, East Carolina University, Greenville, NC.

Workman, R. R. Jr., 1981. Foraminiferal Assemblages of the Nearshore Inner Continental Shelf, Nags Head and Wilmington Areas, North Carolina: Master's Thesis, East Carolina University. 
APPENDIX A: Table of foraminiferal data used to create figures 2 A-D

\begin{tabular}{|c|c|c|c|c|c|c|c|c|c|c|c|c|c|c|c|}
\hline Core/Depth & $\begin{array}{l}\text { Depth } \\
\text { (cm) }\end{array}$ & $\begin{array}{l}\text { Percent } \\
\text { Fossil }\end{array}$ & $\begin{array}{l}\text { Percent } \\
\text { Planktonic }\end{array}$ & Alpha & Species & $\mathrm{N}$ & $\begin{array}{l}\text { E. } \\
\text { excavatum }\end{array}$ & $\begin{array}{l}\text { E. } \\
\text { mexicanum }\end{array}$ & $\begin{array}{l}\text { A. } \\
\text { tepida }\end{array}$ & $\begin{array}{l}\text { A. } \\
\text { parkinsoniana }\end{array}$ & $\begin{array}{l}\text { G. } \\
\text { praegori }\end{array}$ & $\begin{array}{l}\text { R. } \\
\text { floridana }\end{array}$ & $\begin{array}{l}\text { C. } \\
\text { lobatulus }\end{array}$ & Quinqueloculina & $\begin{array}{l}\text { forams } \\
\text { per } \\
\text { gram }\end{array}$ \\
\hline $\begin{array}{l}\mathrm{Y}-60 \mathrm{c17}- \\
20 \mathrm{~cm}\end{array}$ & 19 & $0.0 \%$ & $0.0 \%$ & 9.3 & 22 & 90 & $44.4 \%$ & $5.6 \%$ & $4.4 \%$ & $0.0 \%$ & $12.2 \%$ & $4.4 \%$ & & $6.6 \%$ & 510.46 \\
\hline $\begin{array}{l}\text { Y-60 65- } \\
68 \mathrm{~cm}\end{array}$ & 66 & $5.5 \%$ & $1.0 \%$ & 9.1 & 23 & 104 & $35.9 \%$ & $4.9 \%$ & $8.7 \%$ & $14.6 \%$ & $8.7 \%$ & $2.9 \%$ & & $10.7 \%$ & 442.60 \\
\hline $\begin{array}{l}\text { Y-60 136- } \\
139 \mathrm{~cm}\end{array}$ & 137 & $1.0 \%$ & $0.0 \%$ & 8.1 & 21 & 102 & $32.4 \%$ & $6.9 \%$ & $14.7 \%$ & $11.8 \%$ & $3.9 \%$ & $3.9 \%$ & & $9.80 \%$ & 205.50 \\
\hline $\begin{array}{l}Y-7039- \\
42 \mathrm{~cm}\end{array}$ & 40 & $4.0 \%$ & $0.0 \%$ & 5.5 & 16 & 96 & $16.7 \%$ & $7.3 \%$ & $39.6 \%$ & $10.4 \%$ & $2.1 \%$ & $1.0 \%$ & & $8.3 \%$ & 777.90 \\
\hline $\begin{array}{l}Y-7094- \\
97 \mathrm{~cm}\end{array}$ & 95 & $4.9 \%$ & $0.0 \%$ & 7 & 19 & 98 & $44.9 \%$ & $3.1 \%$ & $0.0 \%$ & $17.3 \%$ & $4.1 \%$ & $2.0 \%$ & & $16.3 \%$ & 521.76 \\
\hline $\begin{array}{l}\mathrm{Y}-70140- \\
143 \mathrm{~cm}\end{array}$ & 141 & $1.1 \%$ & $0.0 \%$ & 5.5 & 16 & 93 & $50.5 \%$ & $3.2 \%$ & $7.5 \%$ & $7.5 \%$ & $9.7 \%$ & $3.2 \%$ & & $2.2 \%$ & 1278.74 \\
\hline $\begin{array}{l}\text { Y-70 223- } \\
226 \mathrm{~cm}\end{array}$ & 224 & $7.7 \%$ & $1.1 \%$ & 7.7 & 20 & 96 & $20.8 \%$ & $11.6 \%$ & $11.6 \%$ & $20.0 \%$ & $4.2 \%$ & $3.2 \%$ & & $12.6 \%$ & 283.25 \\
\hline $\begin{array}{l}Y-918- \\
11 \mathrm{~cm}\end{array}$ & 9 & $0.0 \%$ & $0.0 \%$ & & 3 & 3 & $33.3 \%$ & $0.0 \%$ & $0.0 \%$ & $0.0 \%$ & $0.0 \%$ & $0.0 \%$ & $0.0 \%$ & & 1.02 \\
\hline $\begin{array}{l}Y-9140- \\
43 \mathrm{~cm}\end{array}$ & 41 & $13.1 \%$ & $2.8 \%$ & 3.6 & 73 & 11 & $59.2 \%$ & $0.0 \%$ & $0.0 \%$ & $1.4 \%$ & $21.1 \%$ & $5.6 \%$ & $2.8 \%$ & & 86.89 \\
\hline $\begin{array}{l}Y-9177- \\
80 \mathrm{~cm}\end{array}$ & 78 & $0.9 \%$ & $0.9 \%$ & 4.6 & 112 & 15 & $34.2 \%$ & $1.8 \%$ & $0.0 \%$ & $0.9 \%$ & $19.8 \%$ & $5.4 \%$ & $34.2 \%$ & & 79.88 \\
\hline $\begin{array}{l}Y-10325- \\
28 \mathrm{~cm}\end{array}$ & 26 & $5.1 \%$ & $1.4 \%$ & 8.2 & 19 & 75 & $32.4 \%$ & $6.8 \%$ & $12.2 \%$ & $14.9 \%$ & $8.1 \%$ & $4.1 \%$ & & & 19.40 \\
\hline
\end{tabular}


Appendix B: Table of ALL foraminiferal data for core Y-69

\begin{tabular}{|c|c|c|c|c|c|c|c|c|}
\hline & $\begin{array}{l}17- \\
20 \mathrm{~cm}\end{array}$ & & & $\begin{array}{l}65- \\
68 \mathrm{~cm}\end{array}$ & & & $\begin{array}{l}136- \\
139 \mathrm{~cm} \\
\end{array}$ & \\
\hline Elphidium excavatum & 40 & $44.4 \%$ & Elphidium excavatum & 37 & $35.9 \%$ & Elphidium excavatum & 33 & $32.4 \%$ \\
\hline Gavelinopsis praegori & 11 & $12.2 \%$ & $\begin{array}{l}\text { Ammonia } \\
\text { parkinsoniana }\end{array}$ & 15 & $14.6 \%$ & Ammonia tepida & 15 & $14.7 \%$ \\
\hline Elphidium mexicanum & 5 & $5.6 \%$ & Ammonia tepida & 9 & $8.7 \%$ & $\begin{array}{l}\text { Ammonia } \\
\text { parkinsoniana }\end{array}$ & 12 & $11.8 \%$ \\
\hline Ammonia tepida & 4 & $4.4 \%$ & Gavelinopsis praegori & 9 & $8.7 \%$ & $\begin{array}{l}\text { Elphidium } \\
\text { mexicanum }\end{array}$ & 7 & $6.9 \%$ \\
\hline Bolivina lowmani & 4 & $4.4 \%$ & Elphidium mexicanum & 5 & $4.9 \%$ & Gavelinopsis praegori & 4 & $3.9 \%$ \\
\hline Rosalina floridana & 4 & $4.4 \%$ & $\begin{array}{l}\text { Quinqueloculina } \\
\text { lamarkiana }\end{array}$ & 3 & $2.9 \%$ & $\begin{array}{l}\text { Quinqueloculina } \\
\text { lamarkiana }\end{array}$ & 4 & $3.9 \%$ \\
\hline $\begin{array}{l}\text { Quinqueloculina } \\
\text { lamarkiana }\end{array}$ & 3 & $3.3 \%$ & Rosalina floridana & 3 & $2.9 \%$ & Rosalina floridana & 4 & $3.9 \%$ \\
\hline $\begin{array}{l}\text { Buliminella } \\
\text { elegantissima }\end{array}$ & 2 & $2.2 \%$ & Elphidium gunteri & 2 & $1.9 \%$ & Bucella inusitata & 3 & $2.9 \%$ \\
\hline Cibicides lobatulus & 2 & $2.2 \%$ & Elphidium translucens & 2 & $1.9 \%$ & $\begin{array}{l}\text { Quinqueloculina } \\
\text { seminula }\end{array}$ & 3 & $2.9 \%$ \\
\hline Hanzawaia Strattoni & 2 & $2.2 \%$ & Haynesina germanica & 2 & $1.9 \%$ & $\begin{array}{l}\text { Buliminella } \\
\text { elegantissima }\end{array}$ & 2 & $2.0 \%$ \\
\hline Triloculina triganula & 2 & $2.2 \%$ & $\begin{array}{l}\text { Quinqueloculina } \\
\text { jugosa }\end{array}$ & 2 & $1.9 \%$ & Cibicides fletcheri & 2 & $2.0 \%$ \\
\hline Cibicides fletcheri & 1 & $1.1 \%$ & $\begin{array}{l}\text { Quinqueloculina } \\
\text { seminula }\end{array}$ & 2 & $1.9 \%$ & $\begin{array}{l}\text { Elphidium } \\
\text { translucens }\end{array}$ & 2 & $2.0 \%$ \\
\hline Cibicides sp. & 1 & $1.1 \%$ & Quinqueloculina sm. & 2 & $1.9 \%$ & Haynesina germanica & 2 & $2.0 \%$ \\
\hline Deuteramina ochracea & 1 & $1.1 \%$ & Asteriginata pulchella & 1 & $1.0 \%$ & $\begin{array}{l}\text { Quinqueloculina } \\
\text { poeyana }\end{array}$ & 2 & $2.0 \%$ \\
\hline $\begin{array}{l}\text { Discorbinella } \\
\text { berthelori }\end{array}$ & 1 & $1.1 \%$ & Bolivina lowmani & 1 & $1.0 \%$ & Cibicides lobatulus & 1 & $1.0 \%$ \\
\hline Elphidium gunteri & 1 & $1.1 \%$ & $\begin{array}{l}\text { Buliminella } \\
\text { elegantissima }\end{array}$ & 1 & $1.0 \%$ & Elphidium advenum & 1 & $1.0 \%$ \\
\hline Elphidium sp. & 1 & $1.1 \%$ & Cassidulina sp. & 1 & $1.0 \%$ & Elphidium sp. & 1 & $1.0 \%$ \\
\hline Elphidium translucens & 1 & $1.1 \%$ & Elphidium advenum & 1 & $1.0 \%$ & Nonionella sp. & 1 & $1.0 \%$ \\
\hline Nonionella sp. & 1 & $1.1 \%$ & Hanzawaia Strattoni & 1 & $1.0 \%$ & $\begin{array}{l}\text { Quinqueloculina } \\
\text { bosciana }\end{array}$ & 1 & $1.0 \%$ \\
\hline $\begin{array}{l}\text { Quinqueloculina } \\
\text { frigida }\end{array}$ & 1 & $1.1 \%$ & $\begin{array}{l}\text { Quinqueloculina } \\
\text { bosciana }\end{array}$ & 1 & $1.0 \%$ & Triloculina sp. & 1 & $1.0 \%$ \\
\hline $\begin{array}{l}\text { Quinqueloculina } \\
\text { poeyana }\end{array}$ & 1 & $1.1 \%$ & $\begin{array}{l}\text { Quinqueloculina } \\
\text { poeyana }\end{array}$ & 1 & $1.0 \%$ & Uvigerina auberiana & 1 & $1.0 \%$ \\
\hline \multirow[t]{2}{*}{ Quinqueloculina sp. } & 1 & $1.1 \%$ & Stetsonia minuta & 1 & $1.0 \%$ & & & \\
\hline & & & Triloculina triganula & 1 & $1.0 \%$ & & & \\
\hline Planktonic & 0 & $0.0 \%$ & & 1 & $1.0 \%$ & & 0 & $0.0 \%$ \\
\hline $\begin{array}{l}\text { No. specimens } \\
\text { (modern) }\end{array}$ & 90 & & & 104 & & & 102 & \\
\hline No. species (modern) & 22 & & & 23 & & & 21 & \\
\hline Alpha & 9.3 & & & 9.1 & & & 8.1 & \\
\hline \multirow[t]{3}{*}{ FOSSIL } & & & Ind. Rotalid & 4 & & Hanzawaia sp. & 1 & \\
\hline & & & Elphidium sp.F1 & 1 & & & & \\
\hline & & & Elphidium sp.F2 & 1 & & & & \\
\hline No. specimens (fossil) & 0 & & & 6 & & & 1 & \\
\hline No. species (fossil) & 0 & & & 3 & & & 1 & \\
\hline Percent fossil & 0 & & & $5.5 \%$ & & & $1.0 \%$ & \\
\hline
\end{tabular}


Appendix C: Table of ALL foraminiferal data for core Y-70

\begin{tabular}{|c|c|c|c|c|c|c|c|c|c|c|c|}
\hline & $\begin{array}{l}39- \\
42 c \\
m\end{array}$ & & & $\begin{array}{l}94- \\
97 c \\
m\end{array}$ & & & $\begin{array}{l}140- \\
143 c \\
m\end{array}$ & & & $\begin{array}{l}223- \\
226 c \\
m\end{array}$ & \\
\hline Ammonia tepida & 38 & $\begin{array}{r}39.6 \\
\% \\
\end{array}$ & $\begin{array}{l}\text { Elphidium } \\
\text { excavatum }\end{array}$ & 44 & $\begin{array}{r}44.9 \\
\% \\
\end{array}$ & $\begin{array}{l}\text { Elphidium } \\
\text { excavatum }\end{array}$ & 47 & $\begin{array}{r}50.5 \\
\% \\
\end{array}$ & $\begin{array}{l}\text { Elphidium } \\
\text { excavatum }\end{array}$ & 20 & $\begin{array}{r}20.8 \\
\%\end{array}$ \\
\hline $\begin{array}{l}\text { Elphidium } \\
\text { excavatum }\end{array}$ & 16 & $\begin{array}{r}16.7 \\
\%\end{array}$ & $\begin{array}{l}\text { Ammonia } \\
\text { parkinsoniana }\end{array}$ & 17 & $\begin{array}{r}17.3 \\
\% \\
\end{array}$ & $\begin{array}{l}\text { Gavelinopsis } \\
\text { praegori }\end{array}$ & 9 & $9.7 \%$ & $\begin{array}{l}\text { Ammonia } \\
\text { parkinsoniana }\end{array}$ & 19 & $\begin{array}{r}20.0 \\
\%\end{array}$ \\
\hline $\begin{array}{l}\text { Ammonia } \\
\text { parkinsoniana }\end{array}$ & 10 & $\begin{array}{r}10.4 \\
\% \\
\end{array}$ & $\begin{array}{l}\text { Quinqueloculina } \\
\text { seminula }\end{array}$ & 7 & $7.1 \%$ & $\begin{array}{l}\text { Ammonia } \\
\text { parkinsoniana }\end{array}$ & 7 & $7.5 \%$ & Ammonia tepida & 11 & $\begin{array}{r}11.6 \\
\%\end{array}$ \\
\hline $\begin{array}{l}\text { Elphidium } \\
\text { mexicanum }\end{array}$ & 7 & $7.3 \%$ & $\begin{array}{l}\text { Quinqueloculina } \\
\text { lamarkiana }\end{array}$ & 5 & $5.1 \%$ & Ammonia tepida & 7 & $7.5 \%$ & $\begin{array}{l}\text { Elphidium } \\
\text { mexicanum }\end{array}$ & 11 & $\begin{array}{r}11.6 \\
\%\end{array}$ \\
\hline Bolivina lowmani & 5 & $5.2 \%$ & $\begin{array}{l}\text { Gavelinopsis } \\
\text { praegori }\end{array}$ & 4 & $4.1 \%$ & $\begin{array}{l}\text { Asteriginata } \\
\text { pulchella }\end{array}$ & 3 & $3.2 \%$ & $\begin{array}{l}\text { Elphidium } \\
\text { translucens }\end{array}$ & 6 & $6.3 \%$ \\
\hline $\begin{array}{l}\text { Quinqueloculina } \\
\text { sp. }\end{array}$ & 4 & $4.2 \%$ & Bucella inusitata & 3 & $3.1 \%$ & $\begin{array}{l}\text { Elphidium } \\
\text { mexicanum }\end{array}$ & 3 & $3.2 \%$ & $\begin{array}{l}\text { Quinqueloculina } \\
\text { jugosa }\end{array}$ & 5 & $5.3 \%$ \\
\hline $\begin{array}{l}\text { Hanzawaia } \\
\text { Strattoni } \\
\end{array}$ & 3 & $3.1 \%$ & $\begin{array}{l}\text { Elphidium } \\
\text { mexicanum }\end{array}$ & 3 & $3.1 \%$ & $\begin{array}{l}\text { Elphidium } \\
\text { translucens }\end{array}$ & 3 & $3.2 \%$ & $\begin{array}{l}\text { Gavelinopsis } \\
\text { praegori }\end{array}$ & 4 & $4.2 \%$ \\
\hline $\begin{array}{l}\text { Asteriginata } \\
\text { pulchella }\end{array}$ & 2 & $2.1 \%$ & $\begin{array}{l}\text { Cibicides } \\
\text { fletcheri }\end{array}$ & 2 & $2.0 \%$ & $\begin{array}{l}\text { Rosalina } \\
\text { floridana }\end{array}$ & 3 & $3.2 \%$ & $\begin{array}{l}\text { Rosalina } \\
\text { floridana } \\
\end{array}$ & 3 & $3.2 \%$ \\
\hline $\begin{array}{l}\text { Gavelinopsis } \\
\text { praegori }\end{array}$ & 2 & $2.1 \%$ & $\begin{array}{l}\text { Quinqueloculina } \\
\text { sm. }\end{array}$ & 2 & $2.0 \%$ & $\begin{array}{l}\text { Cibicides } \\
\text { lobatulus }\end{array}$ & 2 & $2.2 \%$ & $\begin{array}{l}\text { Cibicides } \\
\text { fletcheri }\end{array}$ & 2 & $2.1 \%$ \\
\hline $\begin{array}{l}\text { Quinqueloculina } \\
\text { lamarkiana }\end{array}$ & 2 & $2.1 \%$ & $\begin{array}{l}\text { Rosalina } \\
\text { floridana }\end{array}$ & 2 & $2.0 \%$ & $\begin{array}{l}\text { Hanzawaia } \\
\text { Strattoni }\end{array}$ & 2 & $2.2 \%$ & $\begin{array}{l}\text { Cibicides } \\
\text { lobatulus }\end{array}$ & 2 & $2.1 \%$ \\
\hline $\begin{array}{l}\text { Quinqueloculina } \\
\text { poeyana }\end{array}$ & 2 & $2.1 \%$ & $\begin{array}{l}\text { Bolivina } \\
\text { lowmani }\end{array}$ & 1 & $1.0 \%$ & $\begin{array}{l}\text { Quinqueloculina } \\
\text { sm. }\end{array}$ & 2 & $2.2 \%$ & $\begin{array}{l}\text { Quinqueloculina } \\
\text { lamarkiana }\end{array}$ & 2 & $2.1 \%$ \\
\hline $\begin{array}{l}\text { Buliminella } \\
\text { elegantissima }\end{array}$ & 1 & $1.0 \%$ & $\begin{array}{l}\text { Cibicides } \\
\text { lobatulus }\end{array}$ & 1 & $1.0 \%$ & $\begin{array}{l}\text { Bolivina } \\
\text { lowmani }\end{array}$ & 1 & $1.1 \%$ & $\begin{array}{l}\text { Quinqueloculina } \\
\text { sp. }\end{array}$ & 2 & $2.1 \%$ \\
\hline $\begin{array}{l}\text { Elphidium } \\
\text { translucens }\end{array}$ & 1 & $1.0 \%$ & $\begin{array}{l}\text { Elphidium } \\
\text { advenum }\end{array}$ & 1 & $1.0 \%$ & $\begin{array}{l}\text { Buliminella } \\
\text { elegantissima }\end{array}$ & 1 & $1.1 \%$ & $\begin{array}{l}\text { Bolivina } \\
\text { lowmani }\end{array}$ & 1 & $1.1 \%$ \\
\hline Rosalina floridana & 1 & $1.0 \%$ & Elphidium sp. & 1 & $1.0 \%$ & $\begin{array}{l}\text { Haynesina } \\
\text { germanica }\end{array}$ & 1 & $1.1 \%$ & Bucella inusitata & 1 & $1.1 \%$ \\
\hline Sagrina pulchella & 1 & $1.0 \%$ & $\begin{array}{l}\text { Quinqueloculina } \\
\text { jugosa }\end{array}$ & 1 & $1.0 \%$ & $\begin{array}{l}\text { Stetsonia } \\
\text { minuta }\end{array}$ & 1 & $1.1 \%$ & $\begin{array}{l}\text { Deuteramina } \\
\text { ochracea }\end{array}$ & 1 & $1.1 \%$ \\
\hline \multirow[t]{5}{*}{ Bolivina striatula } & 1 & $1.0 \%$ & $\begin{array}{l}\text { Quinqueloculina } \\
\text { poeyana }\end{array}$ & 1 & $1.0 \%$ & $\begin{array}{l}\text { Triloculina } \\
\text { triganula }\end{array}$ & 1 & $1.1 \%$ & $\begin{array}{l}\text { Quinqueloculina } \\
\text { frigida }\end{array}$ & 1 & $1.1 \%$ \\
\hline & & & Triloculina sp. & 1 & $1.0 \%$ & & & & $\begin{array}{l}\text { Quinqueloculina } \\
\text { seminula }\end{array}$ & 1 & $1.1 \%$ \\
\hline & & & $\begin{array}{l}\text { Triloculina } \\
\text { triganula }\end{array}$ & 1 & $1.0 \%$ & & & & $\begin{array}{l}\text { Quinqueloculina } \\
\text { sm. }\end{array}$ & 1 & $1.1 \%$ \\
\hline & & & $\begin{array}{l}\text { Nonionella } \\
\text { atlantica }\end{array}$ & 1 & $1.0 \%$ & & & & Triloculina sp. & 1 & $1.1 \%$ \\
\hline & & & & & & & & & $\begin{array}{l}\text { Lenticulina } \\
\text { Americana }\end{array}$ & 1 & $1.1 \%$ \\
\hline Planktonic & & $0.0 \%$ & & & $0.0 \%$ & & & $0.0 \%$ & & 1 & $1.1 \%$ \\
\hline $\begin{array}{l}\text { No. } \\
\text { Specimens(modern } \\
\text { ) }\end{array}$ & 96 & & & 98 & & & 93 & & & 96 & \\
\hline $\begin{array}{l}\text { No. } \\
\text { Species(modern) }\end{array}$ & 16 & & & 19 & & & 16 & & & 20 & \\
\hline Alpha & 5.5 & & & 7 & & & 5.5 & & & 7.7 & \\
\hline \multicolumn{12}{|l|}{ FOSSILS } \\
\hline Ind. Rotalid & 3 & & Planktonic & 3 & & Ind. Rotalid & 1 & & Cibicides sp. & 4 & \\
\hline \multirow[t]{3}{*}{ Cibicides sp. } & 1 & & Cibicides sp. & 1 & & & & & Bolivina sp. & 2 & \\
\hline & & & Bolivina sp. & 1 & & & & & Ind. Rotalid & 1 & \\
\hline & & & & & & & & & Planktonic & 1 & \\
\hline $\begin{array}{l}\text { No. specimens } \\
\text { (fossil) }\end{array}$ & 4 & & & 5 & & & 1 & & & 8 & \\
\hline No. species (fossil) & 2 & & & 3 & & & 1 & & & 4 & \\
\hline Percent fossil & $4.0 \%$ & & & $4.9 \%$ & & & $1.1 \%$ & & & $7.7 \%$ & \\
\hline
\end{tabular}


Appendix D: Table of ALL foraminiferal data for cores Y-91 and Y-103

\begin{tabular}{|c|c|c|c|c|c|c|c|c|c|c|c|}
\hline \multirow[b]{2}{*}{$\begin{array}{l}\text { Elphidium } \\
\text { excavatum }\end{array}$} & \multicolumn{2}{|c|}{ Y-91 8-11cm } & \multirow[b]{2}{*}{$\begin{array}{l}\text { Elphidium } \\
\text { excavatum }\end{array}$} & \multicolumn{2}{|c|}{ Y-91 40-43cm } & \multirow[b]{2}{*}{$\begin{array}{l}\text { Cibicides } \\
\text { lobatulus }\end{array}$} & \multicolumn{2}{|c|}{ Y-91 77-80cm } & \multirow[b]{2}{*}{$\begin{array}{l}\text { Elphidium } \\
\text { excavatum }\end{array}$} & \multicolumn{2}{|c|}{ Y-103 $25-28 \mathrm{~cm}$} \\
\hline & 1 & $\begin{array}{r}33.3 \\
\%\end{array}$ & & 42 & $\begin{array}{r}59.2 \\
\%\end{array}$ & & 38 & $\begin{array}{r}34.2 \\
\%\end{array}$ & & 24 & $\begin{array}{r}32.4 \\
\%\end{array}$ \\
\hline $\begin{array}{l}\text { Haynesina } \\
\text { germanica }\end{array}$ & 1 & $\begin{array}{r}33.3 \\
\%\end{array}$ & $\begin{array}{l}\text { Gavelinopsis } \\
\text { praegori }\end{array}$ & 15 & $\begin{array}{r}21.1 \\
\%\end{array}$ & $\begin{array}{l}\text { Elphidium } \\
\text { excavatum }\end{array}$ & 27 & $\begin{array}{r}24.3 \\
\%\end{array}$ & $\begin{array}{l}\text { Ammonia } \\
\text { parkinsoniana }\end{array}$ & 11 & $\begin{array}{r}14.9 \\
\%\end{array}$ \\
\hline \multirow[t]{17}{*}{$\begin{array}{l}\text { Rosalina } \\
\text { floridensis }\end{array}$} & 1 & $\begin{array}{r}33.3 \\
\%\end{array}$ & $\begin{array}{l}\text { Rosalina } \\
\text { floridana }\end{array}$ & 4 & $5.6 \%$ & $\begin{array}{l}\text { Gavelinopsis } \\
\text { praegori }\end{array}$ & 22 & $\begin{array}{r}19.8 \\
\%\end{array}$ & $\begin{array}{l}\text { Ammonia } \\
\text { tepida }\end{array}$ & 9 & $\begin{array}{r}12.2 \\
\%\end{array}$ \\
\hline & & & $\begin{array}{l}\text { Cibicides } \\
\text { lobatulus }\end{array}$ & 2 & $2.8 \%$ & $\begin{array}{l}\text { Hanzawaia } \\
\text { Strattoni }\end{array}$ & 6 & $5.4 \%$ & $\begin{array}{l}\text { Gavelinopsis } \\
\text { praegori }\end{array}$ & 6 & $8.1 \%$ \\
\hline & & & $\begin{array}{l}\text { Hanzawaia } \\
\text { Strattoni }\end{array}$ & 2 & $2.8 \%$ & $\begin{array}{l}\text { Rosalina } \\
\text { floridana }\end{array}$ & 6 & $5.4 \%$ & $\begin{array}{l}\text { Elphidium } \\
\text { mexicanum }\end{array}$ & 5 & $6.8 \%$ \\
\hline & & & $\begin{array}{l}\text { Ammonia } \\
\text { parkinsonian } \\
\text { a }\end{array}$ & 1 & $1.4 \%$ & $\begin{array}{l}\text { Elphidium } \\
\text { mexicanum }\end{array}$ & 2 & $1.8 \%$ & $\begin{array}{l}\text { Rosalina } \\
\text { floridana }\end{array}$ & 3 & $4.1 \%$ \\
\hline & & & $\begin{array}{l}\text { Bolivina } \\
\text { lowmani }\end{array}$ & 1 & $1.4 \%$ & $\begin{array}{l}\text { Fursenkoina } \\
\text { fusiformis }\end{array}$ & 2 & $1.8 \%$ & $\begin{array}{l}\text { Hanzawaia } \\
\text { Strattoni }\end{array}$ & 2 & $2.7 \%$ \\
\hline & & & $\begin{array}{l}\text { Elphidium } \\
\text { gunteri }\end{array}$ & 1 & $1.4 \%$ & $\begin{array}{l}\text { Ammonia } \\
\text { parkinsonian } \\
\text { a }\end{array}$ & 1 & $0.9 \%$ & $\begin{array}{l}\text { Quinqueloculin } \\
\text { a lamarkiana }\end{array}$ & 2 & $2.7 \%$ \\
\hline & & & $\begin{array}{l}\text { Sagrina } \\
\text { pulchella }\end{array}$ & 1 & $1.4 \%$ & $\begin{array}{l}\text { Bolivina } \\
\text { lowmani }\end{array}$ & 1 & $0.9 \%$ & $\begin{array}{l}\text { Quinqueloculin } \\
\text { a sm. }\end{array}$ & 2 & $2.7 \%$ \\
\hline & & & $\begin{array}{l}\text { Guttulina } \\
\text { lactea }\end{array}$ & 1 & $1.4 \%$ & $\begin{array}{l}\text { Buliminella } \\
\text { elegantissima }\end{array}$ & 1 & $0.9 \%$ & $\begin{array}{l}\text { Asteriginata } \\
\text { pulchella }\end{array}$ & 1 & $1.4 \%$ \\
\hline & & & $\begin{array}{l}\text { Vasiglobulina } \\
\text { reticulata }\end{array}$ & 1 & $1.4 \%$ & $\begin{array}{l}\text { Elphidium } \\
\text { gunteri }\end{array}$ & 1 & $0.9 \%$ & $\begin{array}{l}\text { Bolivina } \\
\text { lowmani }\end{array}$ & 1 & $1.4 \%$ \\
\hline & & & & & & Elphidium sp. & 1 & $0.9 \%$ & $\begin{array}{l}\text { Bucella } \\
\text { inusitata }\end{array}$ & 1 & $1.4 \%$ \\
\hline & & & & & & $\begin{array}{l}\text { Elphidium } \\
\text { translucens }\end{array}$ & 1 & $0.9 \%$ & $\begin{array}{l}\text { Buliminella } \\
\text { elegantissima }\end{array}$ & 1 & $1.4 \%$ \\
\hline & & & & & & $\begin{array}{l}\text { Haynesina } \\
\text { germanica }\end{array}$ & 1 & $0.9 \%$ & Cibicides sp. & 1 & $1.4 \%$ \\
\hline & & & & & & Bolivina sp.A & 1 & $0.9 \%$ & $\begin{array}{l}\text { Elphidium } \\
\text { advenum }\end{array}$ & 1 & $1.4 \%$ \\
\hline & & & & & & & & & $\begin{array}{l}\text { Quinqueloculin } \\
\text { a seminula }\end{array}$ & 1 & $1.4 \%$ \\
\hline & & & & & & & & & $\begin{array}{l}\text { Quinqueloculin } \\
\text { a sp. }\end{array}$ & 1 & $1.4 \%$ \\
\hline & & & & & & & & & $\begin{array}{l}\text { Valvulineria } \\
\text { laevigata }\end{array}$ & 1 & $1.4 \%$ \\
\hline & & & & & & & & & Bolivina sp. & 1 & $1.4 \%$ \\
\hline Planktonic & 0 & & & 2 & $2.8 \%$ & & 1 & $0.9 \%$ & & 1 & $1.4 \%$ \\
\hline $\begin{array}{l}\text { No. } \\
\text { specimens } \\
\text { (modern) }\end{array}$ & 3 & & & 73 & & & 112 & & & 75 & \\
\hline $\begin{array}{l}\text { No. species } \\
\text { (modern) }\end{array}$ & 3 & & & 11 & & & 15 & & & 19 & \\
\hline Alpha & & & & 3.6 & & & 4.6 & & & 8.2 & \\
\hline \multicolumn{12}{|l|}{ FOSSIL } \\
\hline & & & $\begin{array}{l}\text { Cibicides } \\
\text { lobatulus }\end{array}$ & 5 & & & & & & & \\
\hline & & & ind. Rotalid & 3 & & ind. Rotalid & 1 & & ind. Rotalid & 2 & \\
\hline & & & $\begin{array}{l}\text { Hanzawaia } \\
\text { strattoni }\end{array}$ & 1 & & & & & $\begin{array}{l}\text { Uvigerina } \\
\text { auberiana }\end{array}$ & 2 & \\
\hline & & & $\begin{array}{l}\text { Bucella } \\
\text { inusitata }\end{array}$ & 1 & & & & & & & \\
\hline & & & $\begin{array}{l}\text { Guttulina } \\
\text { austriaca }\end{array}$ & 1 & & & & & & & \\
\hline $\begin{array}{l}\text { No. } \\
\text { specimens } \\
\text { (fossil) }\end{array}$ & 0 & & & 11 & & & 1 & & & 4 & \\
\hline $\begin{array}{l}\text { No. species } \\
\text { (fossil) }\end{array}$ & 0 & & & 5 & & & 1 & & & 2 & \\
\hline $\begin{array}{l}\text { Percent } \\
\text { fossil }\end{array}$ & $0 \%$ & & & $13.1 \%$ & & & $0.9 \%$ & & & $5.1 \%$ & \\
\hline
\end{tabular}




\section{Appendix E: Table of ALL foraminiferal data for core Y-91}

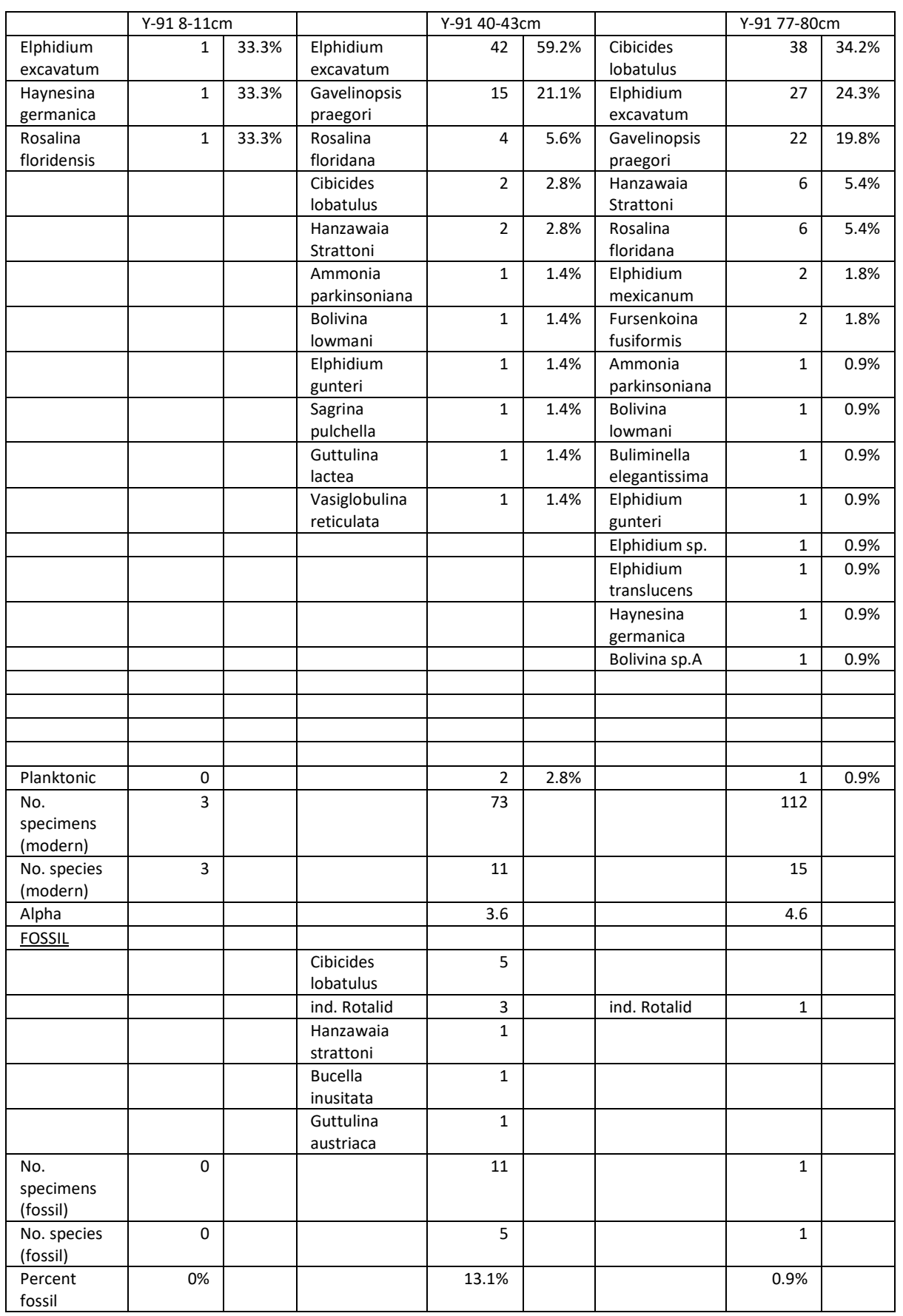




\section{Appendix F: Table of ALL foraminiferal data for core Y-103}

\begin{tabular}{|c|c|c|}
\hline & $\mathrm{Y}-10325-28 \mathrm{~cm}$ & \\
\hline Elphidium excavatum & 24 & $32.4 \%$ \\
\hline Ammonia parkinsoniana & 11 & $14.9 \%$ \\
\hline Ammonia tepida & 9 & $12.2 \%$ \\
\hline Gavelinopsis praegori & 6 & $8.1 \%$ \\
\hline Elphidium mexicanum & 5 & $6.8 \%$ \\
\hline Rosalina floridana & 3 & $4.1 \%$ \\
\hline Hanzawaia Strattoni & 2 & $2.7 \%$ \\
\hline Quinqueloculina lamarkiana & 2 & $2.7 \%$ \\
\hline Quinqueloculina sm. & 2 & $2.7 \%$ \\
\hline Asteriginata pulchella & 1 & $1.4 \%$ \\
\hline Bolivina lowmani & 1 & $1.4 \%$ \\
\hline Bucella inusitata & 1 & $1.4 \%$ \\
\hline Buliminella elegantissima & 1 & $1.4 \%$ \\
\hline Cibicides sp. & 1 & $1.4 \%$ \\
\hline Elphidium advenum & 1 & $1.4 \%$ \\
\hline Quinqueloculina seminula & 1 & $1.4 \%$ \\
\hline Quinqueloculina sp. & 1 & $1.4 \%$ \\
\hline Valvulineria laevigata & 1 & $1.4 \%$ \\
\hline \multirow[t]{6}{*}{ Bolivina sp. } & 1 & $1.4 \%$ \\
\hline & 1 & $1.4 \%$ \\
\hline & 75 & \\
\hline & 19 & \\
\hline & 8.2 & \\
\hline & & \\
\hline ind. Rotalid & 2 & \\
\hline \multirow[t]{5}{*}{ Uvigerina auberiana } & 2 & \\
\hline & & \\
\hline & 4 & \\
\hline & 2 & \\
\hline & $5.1 \%$ & \\
\hline
\end{tabular}


Appendix G: Original core log for Y-69 


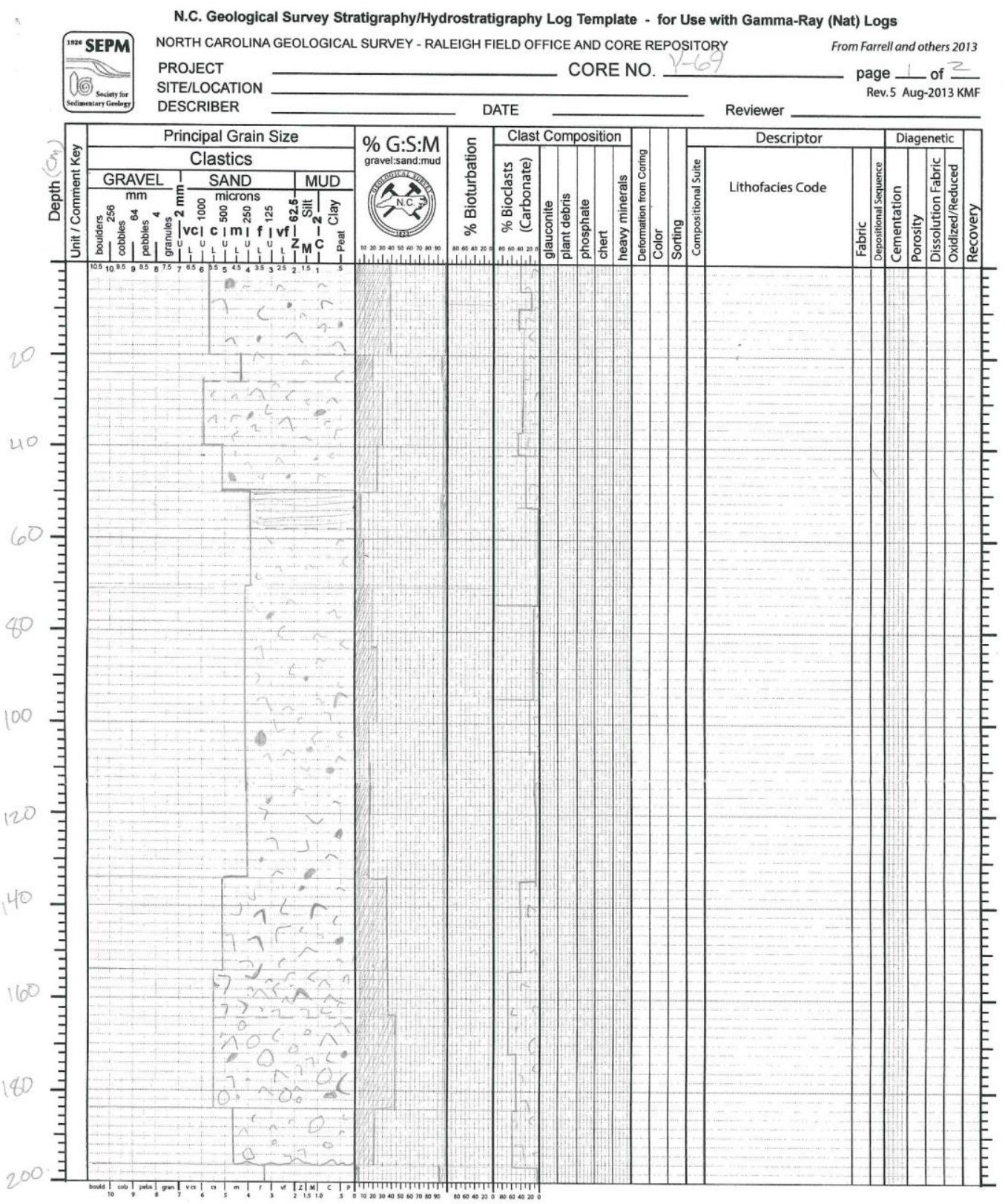

O SEPM 2013 


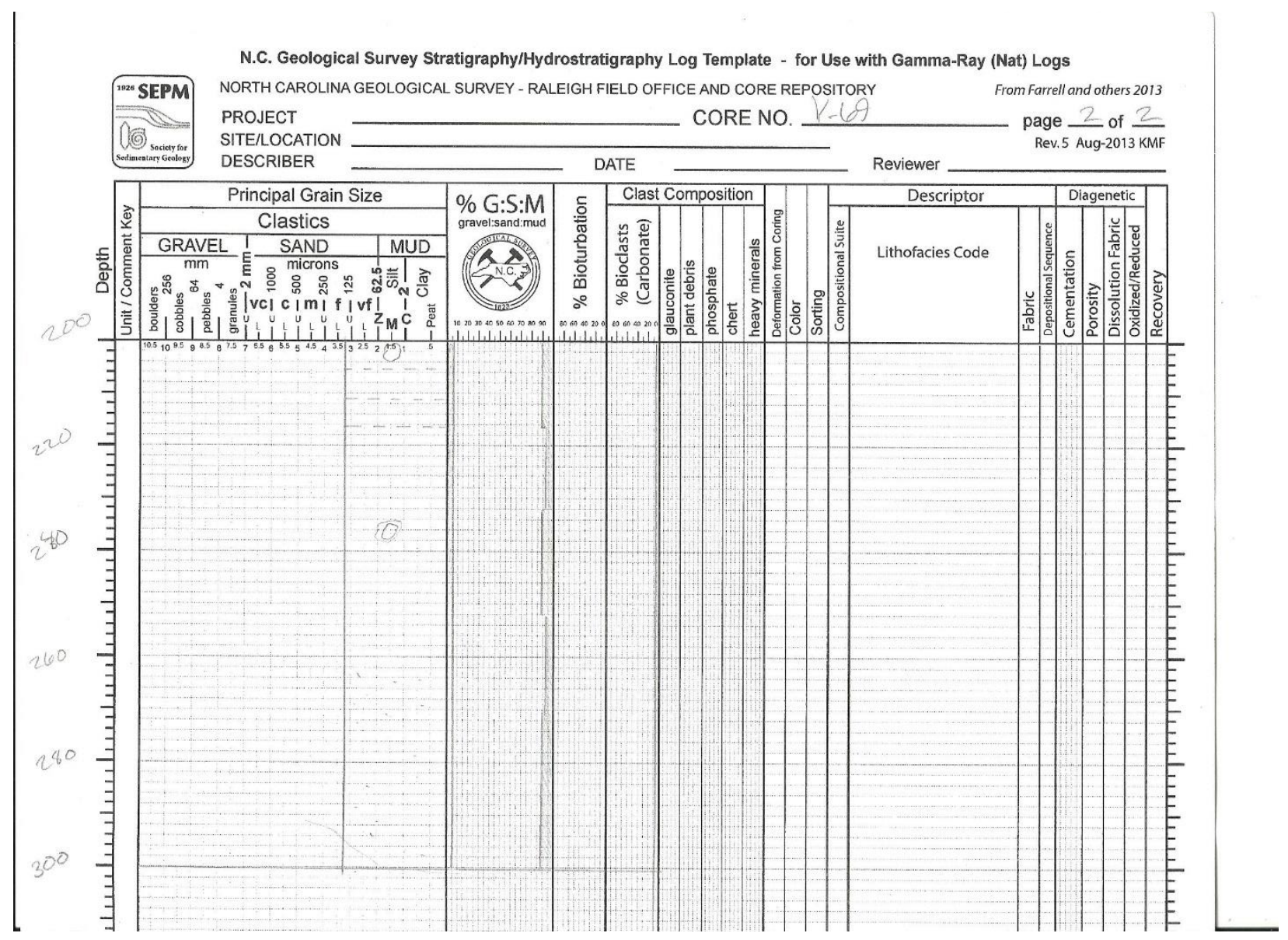


Appendix H: Original core log for Y-70

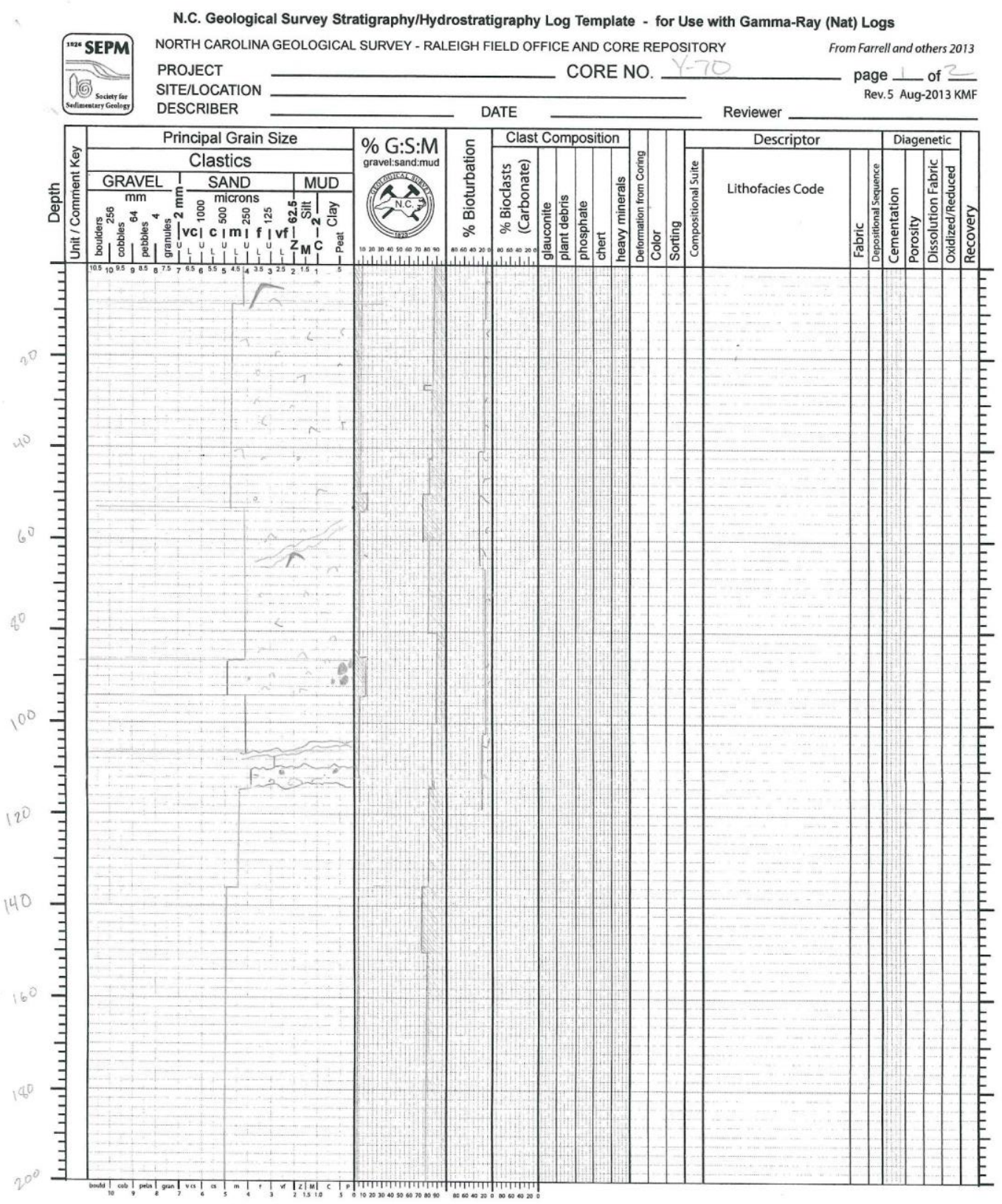

O SEPM 2013 


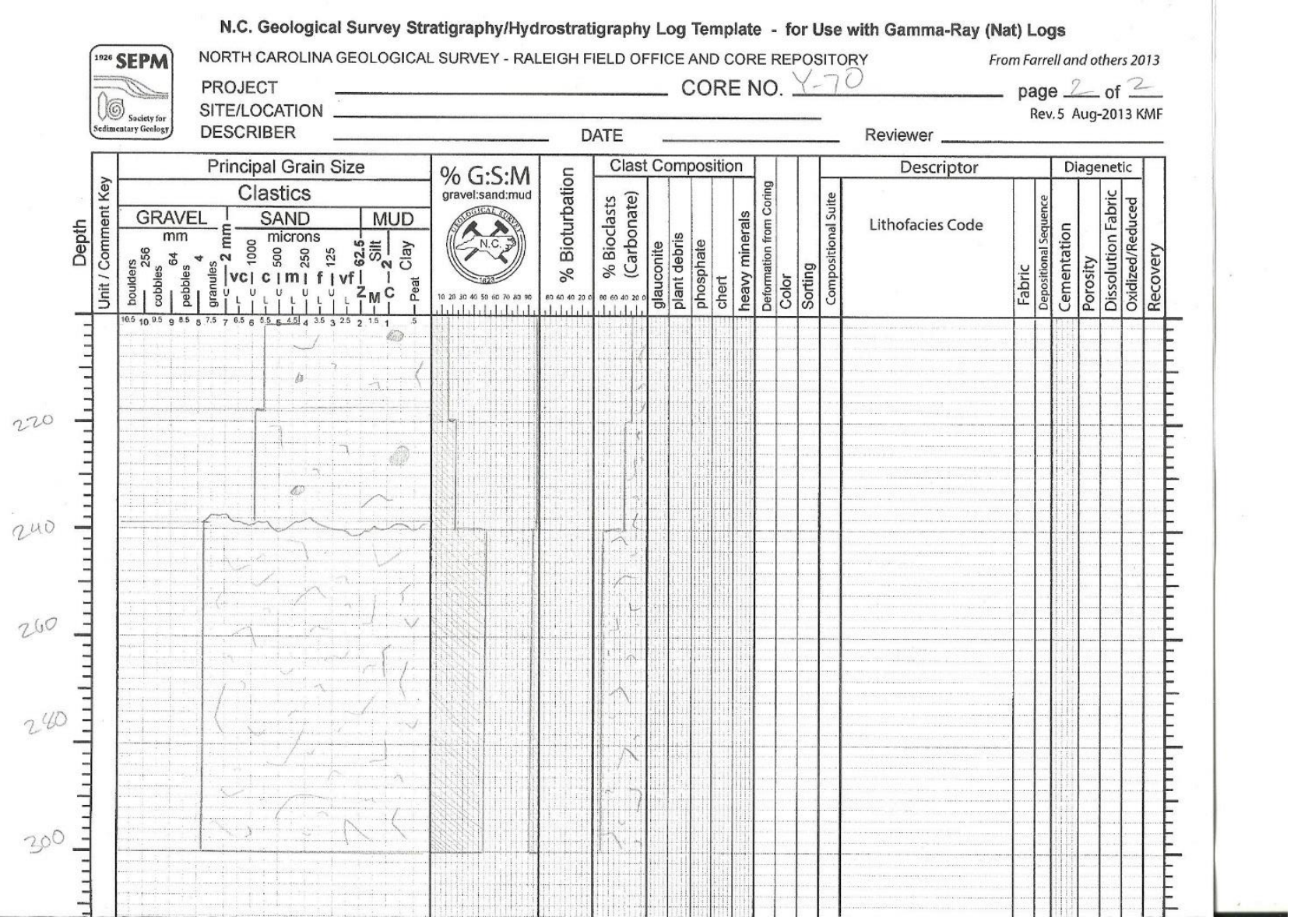




\section{Appendix I: Original core log for Y-91}

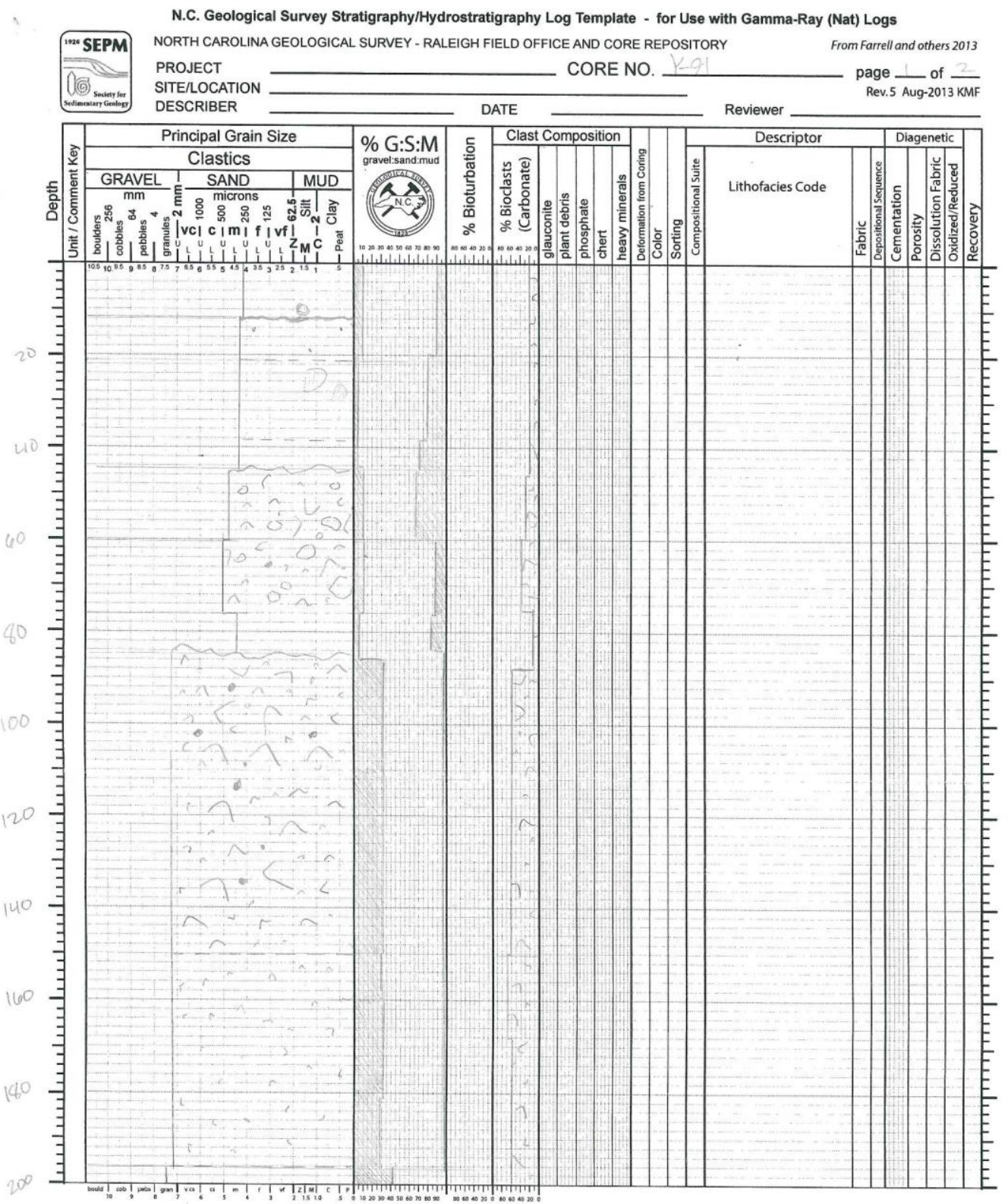

O SEPM 2013 
N.C. Geological Survey Stratigraphy/Hydrostratigraphy Log Template - for Use with Gamma-Ray (Nat) Logs

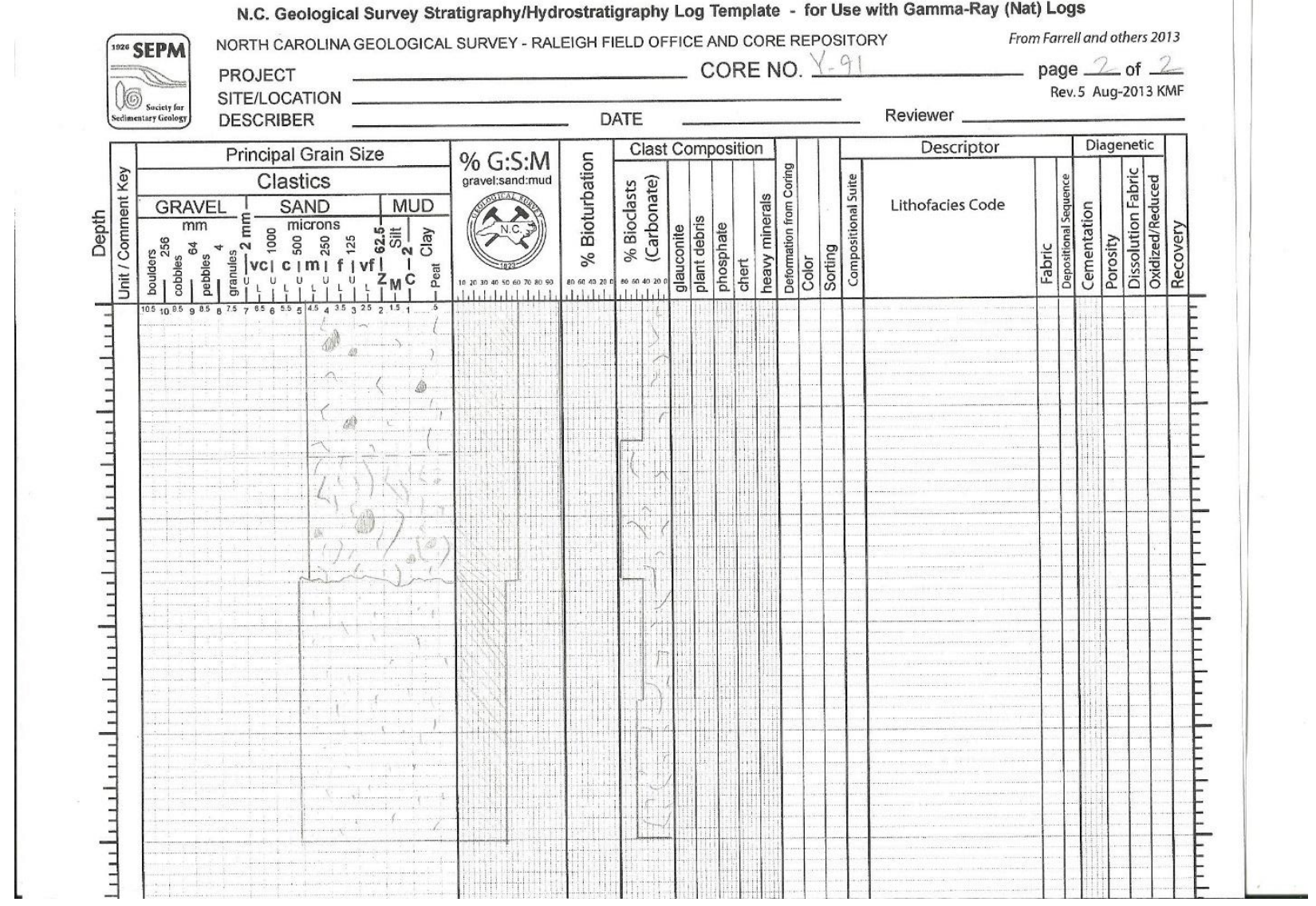




\section{Appendix J: Original core log for Y-103}

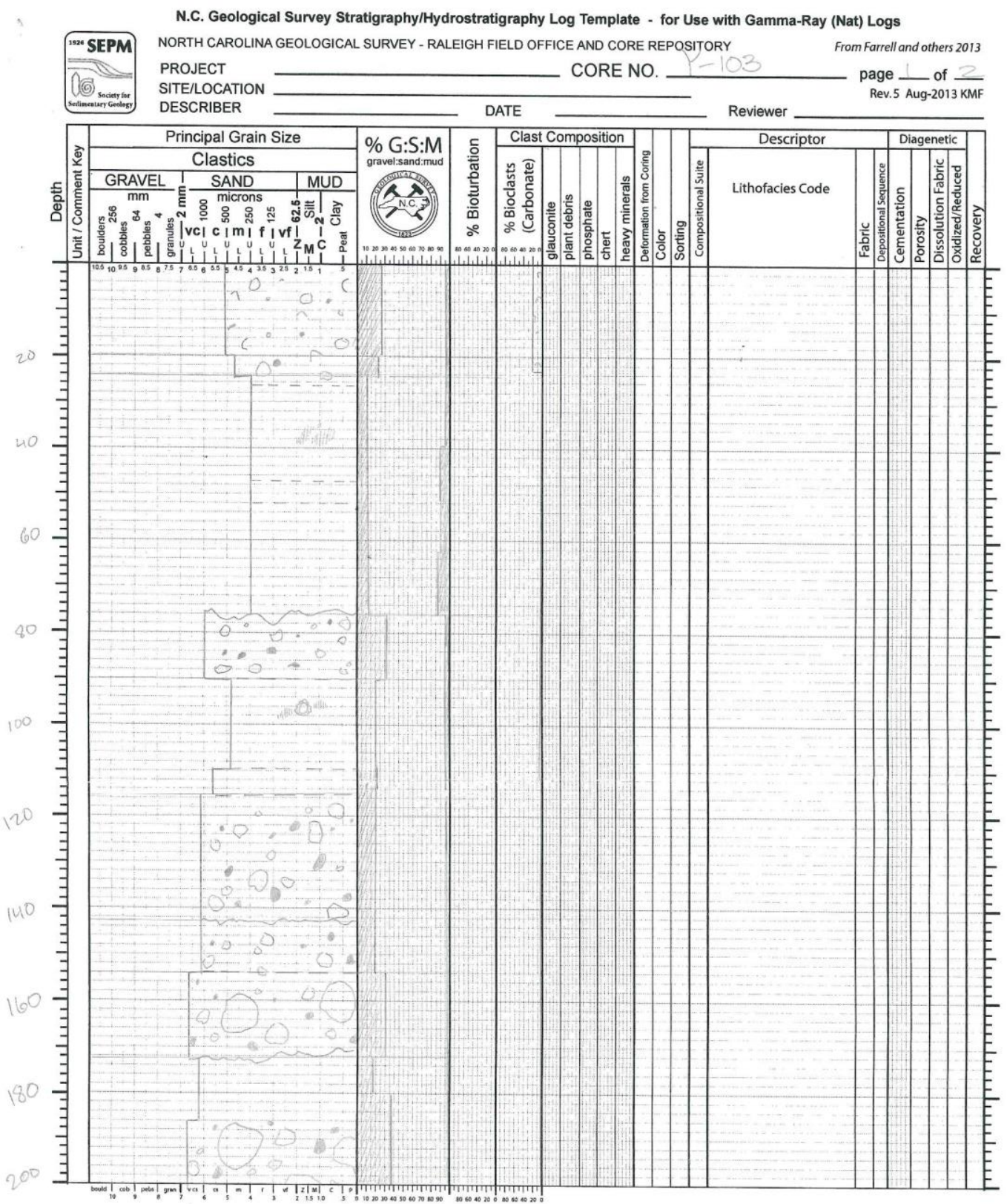

- SEPM 2013 


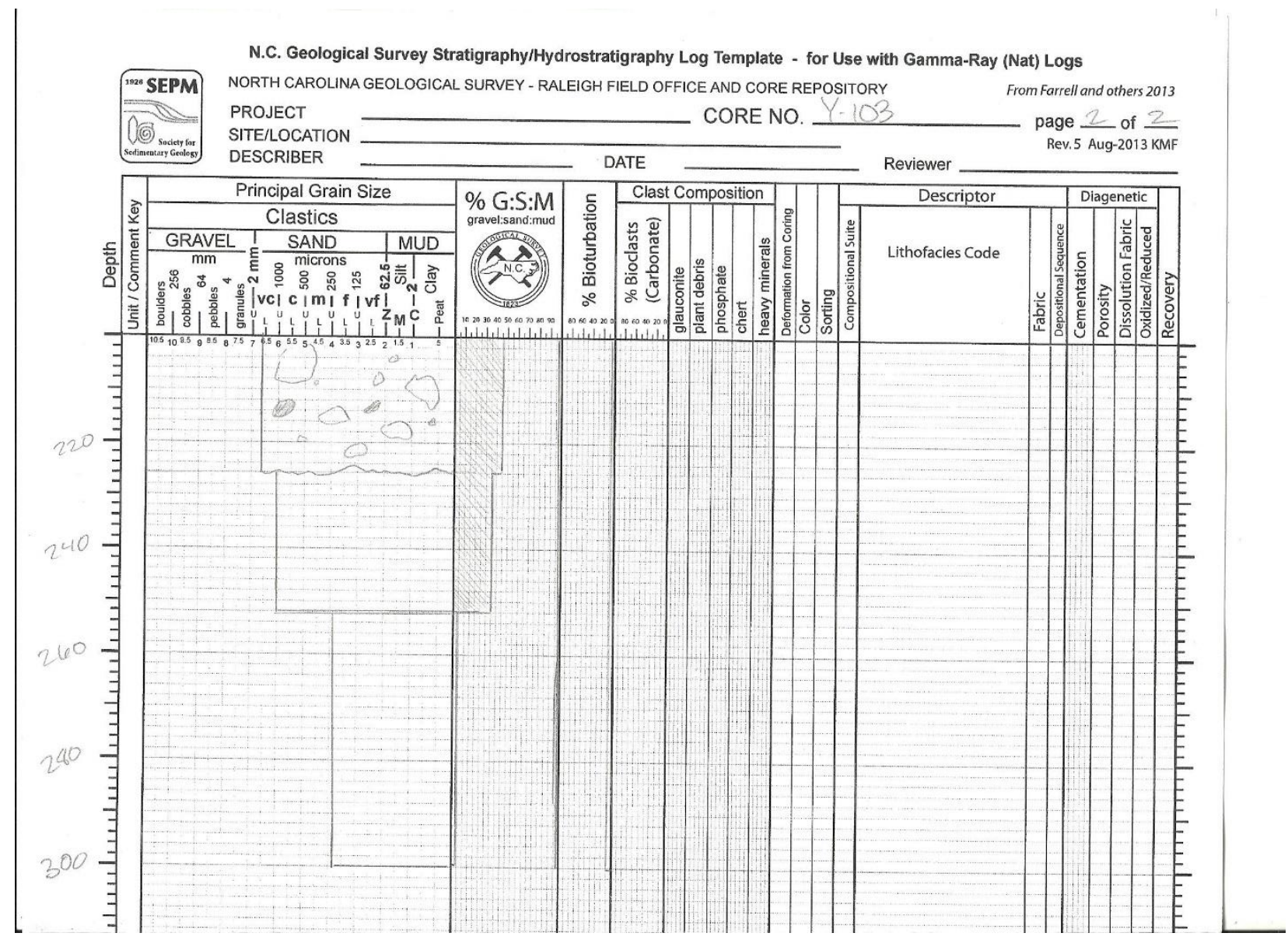

